\title{
Optimizing Click-through in Online Rankings for Partially Anonymous Consumers*
}

\author{
Babur De los Santos ${ }^{\dagger} \quad$ Sergei Koulayev ${ }^{\ddagger}$
}

May 28, 2013

First Version: December 21, 2011

\begin{abstract}
The vast amount of information available online has revolutionized the way firms present consumers with product options. Presenting the best alternatives first reduces search costs associated with a consumer finding the right product. We use novel data on consumer click-stream behavior from a major web-based hotel comparison platform to estimate a random coefficient discrete choice model and propose an optimal ranking tailored to anonymous consumers that differ in their partially revealed price sensitivity. We are able to customize rankings by relating price sensitivity to request parameters, such as the length of stay, number of guests, and day of the week of the stay. In contrast to a myopic popularity-based ranking, our model accounts for the rapidly changing prices that characterize the hotel industry and consumers' search refinement strategies, such as sorting and filtering of product options. We propose a method of determining the hotel ordering that maximizes consumers' click-through rates (CTR) based on the information available to the platform at that time, its assessment of consumers' preferences, and the expected consumer type based on request parameters from the current visit. We find that CTRs almost double when consumers are provided with customized rankings that reflect the price/quality trade-off inferred from the consumer's request parameters. We show that the optimal ranking results in an average consumer welfare 173 percent greater than in the default ranking.
\end{abstract}

Keywords: consumer search, hotel industry, popularity rankings, platform, collaborative filtering, click-through rate, customization, sorting, filtering, search refinement

\footnotetext{
* A previous version of the paper was circulated under the title: "Using Consumer Preferences to Improve upon Popularity Rankings in Online Markets." We thank Elizabeth Honka, Ali Hortaçsu, Joowon Kim, Matthijs Wildenbeest, and participants of the Industrial Organization Society sessions at the Allied Social Sciences Association annual meeting and the Workshop of Switching and Search Costs in Moscow, Russia for helpful comments.

${ }^{\dagger}$ Kelley School of Business, Indiana University, E-mail: babur@indiana.edu.

${ }^{\ddagger}$ Keystone Strategy, E-mail: sergei.koulayev@gmail.com.
} 


\section{Introduction}

In recent years, Internet commerce has achieved significant advances in the reach and complexity of recommendation systems that shape consumer choices in almost all areas of commerce: from looking for product information in online search-engine results to comparing and selecting consumer electronics, books, mortgages, hotel rooms, and flights. ${ }^{1}$ Although the problem of optimal recommendation is not new-salespeople everywhere have long struggled with which product to endorse to a particular consumer - the vast amount of consumer information generated online has revolutionized the way firms collect, analyze, and customize product choices.

At the core of product recommendations is the challenge of matching a set of products to a set of consumers whose tastes are heterogeneous and often unobserved. The accuracy of the match depends on how firms leverage their available information to infer consumer preferences for products. For example, Amazon makes recommendations in diverse product categories by exploiting product correlations present in other customers' transaction histories. Although potential buyers' preferences for a new product are unobservable, the recommendation system is based on the notion that preferences for various products are similar across the set of customers who bought or rated other products in a similar way. ${ }^{2}$

A major challenge for a recommendation system is to serve the category of partially anonymous consumers. Such customers are anonymous to the platform and their past behavior is unobserved (including both their search and clicking behavior) but relevant observable information is available in the current customer visit. For instance, a search request for a hotel room in an online travel platform, which includes the date of stay and the number of guests, reveals whether the consumer is more likely to be a business traveler if the stay is during weekdays, the room request is for single occupancy, and the number of days in advance of the stay the request was made. The premise of this paper is that even though preferences of a particular consumer are unobserved, a significant portion of heterogeneity can be inferred from the request parameters, and can be used to offer a customized

\footnotetext{
${ }^{1}$ See, for example, Ansari and Mela (2003) for a discussion of customization and targeting options available to online sellers.

${ }^{2}$ This is also the underlying assumption of collaborative filtering and other hybrid methodologies would likely improve product rankings and thus reduce search costs for consumers. Examples of these models include Anderson, Ball, Boley, Greene, Howse, Lemire, and Mcgrath (2003); Basilico and Hofmann (2004); Huang, Zeng, and Chen (2004); Melville, Mooney, and Nagarajan (2001); Vozalis and Margaritis (2008); Yu, Schwaighofer, and Tresp (2003). For a nice example of collaborative filtering discussion and application see Moon and Russell (2008).
} 
ranking to the customer. Other information about the consumer that can be used to improve and customize ranking might include location of the user connection, type of browser, IP address, etc. and the consumer's browsing behavior. A recent example of the use of such information is the new ranking strategy of travel site Orbitz, which, after observing that Mac users spend more on hotel rooms, decided to present pricier options to consumers who were searching from a Mac. ${ }^{3}$

In this paper we use highly detailed data on consumer search and click-stream behavior within a major web-based hotel comparison platform to propose an optimal ranking strategy for partially anonymous users. ${ }^{4}$ Platforms such as Orbitz, Kayak, or Travelocity act as intermediaries between hotels and consumers: they collect information about available hotel options and present them in an organized way. ${ }^{5}$ The goal of this paper is to maximize the aggregate click-through rate (CTR) for the presented choices, where a click indicates a consumer's interest and potentially a decision to purchase. ${ }^{6}$

The optimal ranking is derived from a discrete choice model of consumer choice. It models a click on a particular hotel, conditional on the hotel's observed attributes, such as star rating or price, and conditional on the consideration set of hotels observed in the search process. In this framework, the CTR is maximized by ranking products in the order of decreasing expected utility for a given consumer. Indeed, an extant marketing literature on consumer choice has shown that the distribution of heterogeneous tastes plays an important role in the substitution patterns of consumer demand, which are at the core of the recommendation problem. For instance, Ansari et al. (2000), Belanger (2005), Burke (2000), Ricci et al. (2011), and Ghose et al. (2012) use utility based approaches to build recommendations. However methods of estimating attribute weights in the utility ranking vary across these studies. The closest to our approach is work by Ghose et al. (2012), who incorporate user-generated content (e.g., online hotel reviews) into the ranking mechanism.

\footnotetext{
${ }^{3}$ Wall Street Journal, June 26, 2012.

${ }^{4}$ See Bucklin, Lattin, Ansari, Bell, Coupey, Gupta, Little, Mela, Montgomery, and Steckel (2002) for a helpful discussion of research on the Internet using clickstream data.

${ }^{5}$ The influence of these online travel agencies has greatly increased over time. In 2010 almost a half of all non-conference bookings in the United States were made online, and 75 percent were influenced by online search.

${ }^{6} \mathrm{CTR}$ is a standard measure of product relevance, although it is usually complemented with other metrics, such as user reviews. A CTR is defined as the ratio of the number of clicks to the number of impressions (displays) that a product received during a given time period. Although short-term profit objectives may not entirely coincide with click maximization, our ranking is consistent with the long-term success of a search platform, where consumer satisfaction and retention is a key factor.
} 
This study makes two important contributions to the existing literature. First, our ranking exploits information about taste heterogeneity contained in the search parameters, as opposed to a ranking based on the average consumer. This information allows us to categorize consumers into different types and present a customized ranking. We estimate a discrete distribution of unobserved consumer types, and depending on current hotel prices, an optimal ranking is chosen that targets one of these types. Because CTR is non-linear in the unobserved component, the optimal ranking does not necessarily target the median type. For instance, prioritizing more expensive, higherquality products may result in click gains among quality-centered consumer types that do not outweigh expected click losses among price-sensitive consumer types.

Second, we explicitly account for the endogeneity of search refinements on the ranking, such as sorting and filtering of search results, when deriving the optimal ranking. Search preference is an important factor, as the composition of consideration sets is a product of both the default product ranking and user search refinements. ${ }^{7}$ For example, searchers who sort hotels by increasing price tend to observe cheaper hotels that are further away from city center. If the proportion of such searchers is substantial, one may improve the diversity of choice sets by giving prominence to hotels closer to city center or of higher quality. As future search refinements are unobserved, we evaluate a candidate ranking by integrating over potential search strategies. ${ }^{8}$ Recognizing the endogeneity of search on the optimal ranking problem, we additionally estimate a model of consumer search where both click and search are jointly determined. Using counterfactual experiments, we show that a ranking obtained through a simpler, static discrete choice model achieves 70 percent of the gain obtained through the search model.

The optimal ranking leads to an almost twofold increase in CTR compared to the default ranking used on the platform at the time of data collection. While the exact formula used by the platform is proprietary, its primary component is a hotel's past popularity, measured by the

\footnotetext{
${ }^{7}$ Firms' recognition of the importance of consumer search behavior is evident in managers' accurate sense of consumer search and patronage behavior (Urbany, 2002), in auctions for prominent positions in sponsored online search advertising, and expenditures on search engine optimization to improve firms' position in search results (Berman and Katona, 2011). Similar firm actions to improve product visibility can be seen in supermarket shelving fees, fees for featured listings on recommendation web sites, and online price comparison engines. Consumer behavior in other settings exemplifies that the way information is presented to consumers matters. For example, Ho and Imai (2006) find that being listed first on a voting ballot increases a candidate's vote share.

${ }^{8}$ The default ranking is presented in response to the initial search request, and before any search refinement, such as sorting or filtering. Potentially, it is possible to further customize rankings for filtered searches. We leave this possibility for future research.
} 
number of clicks the hotel received during a specified time window. Although intuitively appealing, the popularity-based ranking has two major drawbacks. First, it fails to adjust to changes in current prices, which is particularly problematic within the hotel industry, where prices change frequently due to demand fluctuations and revenue management models. ${ }^{9}$ Second, the popularity ranking suffers from a feedback effect which causes the ranking to persist over time. As a result, previous rankings may distort future consumer choices. ${ }^{10}$ In contrast, rankings based on a structural model of click have an immediate advantage of automatic adjustments to changing market conditions: the hotel's utility is re-evaluated for each consumer request at current prices and availability. An important feature of our ranking is its feasibility. All required data can be collected within the platform. Further, because preference parameters are pre-computed, a customized ranking that takes into account current prices can be presented in response to the consumer's initial request.

\section{Data}

The data consist of consumer search histories for hotel rooms on a major web-based hotel comparison platform. A search history starts with a search request. The request includes city, dates of stay, number of people, and number of rooms. Following the request, the website presents the consumer with all hotels in the city with available rooms that satisfy the request parameters. The hotel options are organized in pages with 15 hotels per page, displaying hotel name, price, as well as various non-price characteristics: star rating, neighborhood, distance to city center, proximity to airports, and so on. All consumers are presented with a first page of results upon submitting the search request. They can either click on a hotel on that page or continue browsing the next page. Although there are many ways to search, there are two general categories of search strategies. First, one may page through the default results. Second, consumers may employ other search refinement

\footnotetext{
${ }^{9}$ The underlying reason for the under-performance of popularity-based rankings is data sparsity: frequent price changes do not permit the collection of enough data to evaluate a hotel's popularity at a new price level. This is similar to the problem of valuation of new products and is sometimes called a "cold start problem."

${ }^{10}$ The primary explanation is limited search: in our data, top-placed hotels collect almost 20 percent of all clicks. Numerous studies have documented this phenomenon. By tracking eye movements, Lohse (1997) finds that yellow page advertisements near the section heading are more likely to catch a reader's attention. Using Google search results, Joachims et al. (2005) show that consumers are more likely to click on items ranked high on the list. Ghose and Yang (2009) find that sponsored search advertisements have higher CTRs when located at a more prominent position in the ranking. As Hendricks, Sorensen and Wiseman (2010) point out, in these situations the superior alternatives may be ignored if placed in worse positions in the ranking, while consumers waste time evaluating inferior products that appear in better positions. These findings suggest that the order in which options are presented greatly influences consumer choices.
} 
tools such as sorting or filtering results based on hotels' characteristics. In our data, we observe the listing of all hotels displayed to the consumer as a result of their search request and all subsequent sorting or filtering.

We also observe which hotel the consumer clicked as part of their search process. About half of searchers who click do so only once, in which case this is the end of the search session. If more than one click is made, we consider the last click as the hotel that is the closest match to the user's preferences among the searched hotels. Alternatively, the user can leave the website without clicking.

The platform is a search aggregator, which compiles and presents hotel information from other travel websites, but it does not offer hotel bookings. After clicking on a hotel option, the user is redirected to another website where a booking can be made. A potential limitation of the data is that we do not observe whether two sessions were made by the same person (a session is a set of search requests within 24 hours of each other). Therefore, we conduct our analysis as if every search were made by a unique individual. Below we discuss these issues in greater length.

In total, the sample contains 23,959 unique search histories for Chicago, by consumers who searched the platform during May 1-31, 2007. In total 148 Chicago hotels were available for online booking during that period. A search typically returns 130 to 140 hotel options, depending on availability. This wealth of options creates a non-trivial search problem for a consumer. Hotels in the Chicago market include those in the city of Chicago itself, the satellite towns (Evanston, Skokie, etc.), as well as those in close proximity to airports (O'Hare and Midway). There are also various neighborhoods within the city center. These geographical parameters are observed by consumers during search.

Table 1 summarizes our data. On average, consumers in our sample search for a hotel room 33.5 days in advance of their stay, 60 percent of them stay over a weekend, and the average number of guests is 1.84. The hotels in the dataset show significant price variation. The range of prices in the sample is from $\$ 16$ to $\$ 1,500$, with an average of $\$ 230$. This reflects both cross-sectional as well as temporal variation in prices (below we document price variation in greater detail). The variation in contents of the first page can be summarized by the percentage of observations in which a given hotel appeared on the first page. In the sample, an average hotel appears on the first page about 30 percent of the time, with substantial heterogeneity: some hotels never appear on the first page, 
while others appear there every second search or more.

In the sample 33 percent of the search requests ended in a click. Of these, more than half on the first page. An individual hotel's CTR ranges between 1.1 and 5.8 percent. Prices of hotels that received a click are somewhat lower, which is expected, as clicks can be considered revealed preferences. There is considerable variation in search behavior among consumers, which takes the form of a long tail on hotel choices. An average consumer observes two pages of results (30 hotels). Table 2 presents the breakdown of the different search refinement strategies of the consumers in the sample. We find that 40.8 percent of the consumers never search-they only observe the first page of results. Another 16.8 percent of visitors continue browsing past page 2 of the hotel listings. Almost three times as many consumers, 42.4 percent of all, instead choose to refine search results, and contribute a disproportionately higher share of clicks, 48 percent. These facts suggest that consumers do not find the default ranking system very helpful.

Finally, Figure 1 illustrates the extent of within-page search. Although we do not observe this search directly, indirect evidence can be obtained from the distribution of clicks across hotel position (ranging from 1 to 15 on the page of results). Of all clicks on the first page, 22 percent went to the hotel in position 1 , in contrast with 11 percent to position 2 , and 3 percent to the last

position on the page. A similar pattern exists for other pages: overall 19 percent of the clicks were for a hotel located at the top of any page.

Next, we analyze further the characteristics of the current ranking.

\subsection{Default Popularity Rankings}

Previously we have seen that consumers who only browse the default hotel ranking contribute a smaller share of clicks than consumers who refine their search, a finding that questions the relevance of the default ranking system. Specifically, we want to know whether the current information available to the search engine - parameters of user request and current hotel prices - affects a hotel's display prominence. We test these hypotheses using regression analysis of pairwise hotel rankings. We create all possible hotel pairs from the hotel listings ranked by the default criteria. The resulting dataset consists of 9,734 hotel pairs and over 3 million observations of these pairs over time. For each hotel within a pair, we observe the page index, the rank (position on the page) and the price. For every search $s$ we define an indicator variable $Y_{j k s}$ of whether hotel $j$ is ranked higher than hotel 
$k, Y_{j k s}=1$ position $_{j s}<$ position $\left._{k s}\right)$. By considering hotel pairs, rather than hotels in isolation, we eliminate contemporaneous shocks that affect both hotels' chances for prominence.

Table A-1 presents results of regression of $Y_{j k s}$ on request parameters (weekend stay, number of people, advance search) as well as differences in the prices of the two hotels. The price difference between hotels has a negligible effect on relative ranking: if hotel $j$ becomes relatively more expensive than hotel $k$ by $\$ 100$, the probability of hotel $j$ being in a lower position increases by 6 percent. This does not reflect consumer preferences: other things equal, lower-priced hotels should instead have better rankings, as they are more attractive to consumers. Similarly, parameters of search request have little explanatory power for pairwise rankings.

To summarize, the data rejects the hypothesis that the search engine is using current information to rank hotels. Instead, it appears that the ranking is based on a measure of past hotel popularity (a fact confirmed by the website's managers). As does any ranking method based on past consumer choice data, the popularity ranking suffers from a feedback effect: hotels that initially obtained prominence will continue to enjoy it, even though their actual attractiveness may change. As a result of this persistence, consumers ignore superior options or waste resources learning about inferior ones, a phenomenon often referred to as a bad-herding behavior. ${ }^{11}$ In contrast, the optimal ranking is based on current information available to the search engine. As we find below, the result is a dramatic improvement in relevancy of displayed hotels. ${ }^{12}$

\footnotetext{
${ }^{11}$ See for example Hendricks, Sorensen and Wiseman (2010) for a model of the effects of observational learning on herding behavior. Collaborative-filtering algorithms have been proposed extensively in the literature, e.g. Hofmann (1999), Billsus and Pazzani (1998), Kim (2001), Canny (2002) and Basilico and Hofmann(2004).

${ }^{12}$ In order to formalize this discussion we also simulate the effects of a popularity ranking based on observed consumer search and click behavior. Because we know how much consumers search, we are able to quantify the feedback that affects a popularity ranking. First, we obtain the probability distribution of search strategies among users of the platform. In other words, for a random consumer visiting the platform, we estimate the probability that the user will use a particular search strategy and hence observe a particular set of hotels. For 19,898 search sessions in our sample, given the user choice of a search strategy which affects the results displayed, we are able to generate counterfactual choice sets that would have been obtained under an alternative ranking and a vector of hotel prices. Second, we choose an initial ranking of hotels, using the actual choice sets observed by consumers who visited the platform. We compute the average predicted click probability for every hotel and create a popularity-based ranking. Third, we simulate choice sets that would have been observed by consumers who visited the platform. These choice sets depend not only on the initial sorting but also on hotel prices.

The simulation shows that the top ranked hotels are likely to remain there and the hotels that were initially disfavored get little chance to improve their rating. Of the five hotels that were initially placed on top, four remain at one of the top 5 positions for the rest of the month even though there were significant price changes throughout. The top hotel increases from a click probability of 6 percent on the first day to a maximum of 14 percent and decreases to 4 percent on the last day. The click probability of the fourth hotel increases steadily from 3 percent to 35 percent. It is notable how fast popularity drops as we go down the list, even within the first page of results. Only a few hotels in the first page and top positions have significant popularity rankings. For those hotels on the second page, both the level and the change in ranking are significantly smaller.
} 


\section{$3 \quad$ A Model of Optimal Ranking}

We assume that the objective of the ranking system is to maximize the CTR on the organic set of search results within a product comparison website. Although, in general, this goal does not coincide with profit maximization, except for the case where all revenues are derived from click fees and the fees are constant across products. We consider click maximization as an important input into the overall profit maximization problem of a platform. For instance, there are ways to earn additional revenues without distorting organic search results, such as through clearly marked sponsored offerings.

We use a discrete choice modeling approach to managing CTR on a search platform. After a user compares the attractiveness of the options observed during the search session the click is an outcome of optimal consumer choice. In this case, there are two major dimensions of heterogeneity among consumers that together determine a click. First, consumers have idiosyncratic tastes for hotels, and in particular they differ in willingness to pay for higher quality (closer to city center, higher rating, better neighborhood). A portion of this heterogeneity can be linked to observable parameters, such as whether the user is staying over the weekend. However, a substantial component of heterogeneity is likely to remain unobserved. Second, consumers differ in their search strategies: users who employ various sorting and filtering search tools will explore additional hotels that "passive" users will not observe. Both types of strategies are inherently connected: click and search decisions are both outcomes of consumer behavior. Consequently, any ranking might in turn affect search behavior. In the next sections we will abstract from this issue by assuming that search refinements are exogenously given. This assumption is made for the sake of practicality of the optimal ranking method. As a robustness check, in Section 6 we estimate a model of endogenous search and find that our assumption does little to alter the effectiveness of the ranking.

In this section we introduce the consumer's choice problem represented by their click decisions. Then we propose a couple of simplified examples to illustrate two key issues that a ranking must

address: heterogeneity in unobserved tastes and differences in ranking when users employ sorting and filtering tools. These examples allow us to develop intuition as to why and how a ranking that targets a median consumer will not achieve the same results as a ranking based on distributional parameters. Finally, we generalize these examples to propose a step by step ranking algorithm that 
maximizes CTR.

\subsection{Consumer's Choice Problem}

We adopt a revealed preference approach to explaining clicks: a click on a particular hotel indicates a preference toward that hotel among other alternatives in the choice set, plus the outside option. ${ }^{13}$ Let $i$ stand for the index of an individual consumer, and $j$ the index of a hotel. The utility of consumer $i$ from clicking on hotel $j$ is given by:

$$
u_{i j}=-\alpha_{i} p_{i j}+\beta_{i}^{\prime} X_{j}+\delta L_{i j}+\varepsilon_{i j}
$$

where $p_{i j}$ is the hotel's price as observed by consumer $i$, and $X_{j}$ is a vector of non-price hotel characteristics: star rating, distance to city center, distance to airport, neighborhood, brand, etc. Parameter $\alpha_{i}$ measures price sensitivity, while $\beta_{i}$ is a vector of tastes for non-price characteristics. The parameter $\delta$ measures the effect of the hotel's position on the display, $L_{i j}=1, \ldots, 15$, on the probability of clicking. Recall from Figure 1 that on average consumers are more likely to click on hotels located at higher positions. Lastly, $\varepsilon_{i j}$ are i.i.d. Type I extreme value error terms that represent the consumer's idiosyncratic preferences for a particular hotel.

The utility of the outside option is given by

$$
u_{i 0}=\gamma_{0}+\gamma_{1}^{\prime} R_{i}+\varepsilon_{i 0}
$$

where $\gamma_{1}$ is a vector of parameters that captures differences in propensity to click among users with different parameters of request, $R_{i}$. These include dates of search and dates of stay, from which we can derive advance purchase. For example, a consumer who is searching further in advance might have a higher value for the outside option, due to the possibility of searching later.

Taste heterogeneity in our model arises due to differences in parameters of request, as well as

\footnotetext{
${ }^{13}$ Since a click does not necessarily result in a booking (and we do not observe bookings), we interpret the utility of clicking as the expected value which takes into account the possibility that the booking may not occur. For example, the consumer may find new information on the hotel's website that was not available on the search website.
} 
unobserved heterogeneity:

$$
\begin{aligned}
& \beta_{i}=\beta_{0}+\beta_{1}^{\prime} R_{i} \\
& \alpha_{i}=\alpha_{0 i}+\alpha_{1}^{\prime} R_{i}
\end{aligned}
$$

where $\beta_{0}$ is a vector of baseline tastes, and $\beta_{1}$ is a matrix of interaction terms. In addition to the observable heterogeneity, our model for the price coefficient includes a random shock $\alpha_{0 i}$, with a log-normal distribution.

Let $C_{i}$ be the consumer's choice set, a collection of hotels discovered during the search process, and denote by $P_{i}$ the vector of current hotel prices. Utility maximization implies that we should observe a consumer $i$ clicking on hotel $j \in C_{i}$ if and only if $u_{i j}>u_{i k}, k \neq j, k \in C_{i}$. Conditional on the observed data, the probability that a random consumer would click on hotel $j$ is:

$$
P\left(j \mid C_{i}, P_{i}, R_{i}\right)=\int \frac{\exp \left(-\alpha_{i} p_{i j}+\beta_{i}^{\prime} X_{j}+\delta L_{i j}\right)}{\exp \left(\gamma_{0}+\gamma_{1}^{\prime} R_{i}\right)+\sum_{k \in C_{i}} \exp \left(-\alpha_{i} p_{i k}+\beta_{i}^{\prime} X_{k}+\delta L_{i j}\right)} d F\left(\alpha_{i}\right)
$$

In this expression, $F\left(\alpha_{i}\right)$ is the probability distribution of consumer types in the population. From (5), the CTR - probability that a consumer will click on any hotel in her choice set - is found as:

$$
\begin{aligned}
C T R\left(C_{i}, P_{i}, R_{i}\right) & =\sum_{j \in C_{i}} P\left(j \mid C_{i}, P_{i}, R_{i}\right) \\
& =\int \frac{\sum_{k \in C_{i}} \exp \left(\mu_{i j}\right)}{\exp \left(\mu_{i 0}\right)+\sum_{k \in C_{i}} \exp \left(\mu_{i k}\right)} d F\left(\alpha_{i}\right)
\end{aligned}
$$

where $\mu_{i j}=-\alpha_{i} p_{i j}+\beta_{i}^{\prime} X_{j}+\delta L_{i j}$ and $\mu_{i 0}=\gamma_{0}+\gamma_{1}^{\prime} R_{i}$ are obtained from (1). This formula highlights the purpose of the ranking system, which is to shape the contents of consumer choice set, $C_{i}$, in a way that maximizes CTR. Next we consider the general maximization problem and suggest a couple of examples to illustrate the roles of consumer preferences across different model specifications.

\subsection{Optimal Platform Ranking}

In this section, we outline the optimal platform ranking that coincides with a consumer utility maximization problem. In this case ranking will be presented by declining expected utility for a 
given consumer. However, in this section rather than sorting products by mean utility, we will exploit distributional parameters of two dimensions of heterogeneity to customize the offering to consumers. We do this in two steps.

First we account for consumers' idiosyncratic tastes for hotels, in particular their differences in willingness to pay for quality (which might be a combination of different hotel attributes: closer to city center, higher rating, better neighborhood). Some of that heterogeneity can be linked to observable parameters, such as whether the user is staying over the weekend, the number of days in advance the hotel is booked and whether the consumer is searching for single occupancy. All this observable parameters contain information about preferences and particular about the quality/price tradeoff. However, a substantial component of heterogeneity is likely to remain unobserved.

Second, consumers differ in their search preferences: users who employ various sorting and filtering search tools will explore additional hotels that "passive" users will not observe. By referring to search patterns as "preferences", we will implicitly assume that they are exogenously given. In reality, search refinements will depend on the choice of ranking, to the extent that search refinements are rational decisions made by a consumer. Unfortunately, an appropriate account for endogeneity of search decision makes the ranking method unfeasible: estimation of search models requires specialized skills not available to managers of most web platforms. As a robustness check, in Section 6 we estimate such a model and find that a ranking based on exogenous search achieves $70 \%$ of the gain brought by a fully optimal ranking.

In what follows, we begin by introducing a general formulation of the optimal ranking under unobserved utility and search preferences. Then, we develop simple examples that illustrate why and how a ranking that targets a median consumer will fall short of results provided by our ranking.

\subsection{Ranking algorithm for heterogeneous preferences}

In section 3.1 we observed that the outcome of a discrete choice model of click is $C T R\left(C_{i}, P_{i}, R_{i}\right)-$ expected click rate among consumers with common request parameters $R_{i}$, choice set $C_{i}$ and vector of current prices, $P_{i}$. Conditional on these variables, consumers are observationally equivalent, or, in other words, partially anonymous. At the time when the ranking is presented, the search engine "observes" $P_{i}$ and $R_{i}$, but not $C_{i}$, as the latter depends on consumer search refinements, which are not yet made. Therefore, $C_{i}$ must be integrated out to construct a computable objective function. 
Let $\mathbf{r}_{i}=\left(r_{i 1}, \ldots, r_{i N}\right)$ be the ranked vector of $N$ hotels, as presented to user $i$. Let $h \in H$ be a search strategy among a set of potential strategies $H$. Given $\left(P_{i}, \mathbf{r}_{i}\right)$, define a mapping from a search strategy $h$ to a choice set $C_{i}$ :

$$
C_{i}=\mathbf{C}\left(h, P_{i}, \mathbf{r}_{i}\right)
$$

This mapping formalizes the relationship between the ranking and consumer search refinement strategies in shaping the choice set. Its properties are dictated by the specifics of a given search platform (e.g. search tools available, user interface, etc.), as well as the availability of hotel options. To reduce the computational burden, we simplify the mapping function (8) by excluding interactions between $h_{i}$ and $\mathbf{r}_{i}$. Specifically, we assume an additive structure:

$$
\mathbf{C}\left(h_{i}, P_{i}, \mathbf{r}_{i}\right)=D_{1}\left(\mathbf{r}_{i}\right)+\ldots+D_{k}\left(\mathbf{r}_{i}\right)+\tilde{\mathbf{C}}\left(h_{i}, P_{i}\right)
$$

Accordingly, every search history $h$ has two distinct stages. At the initial stage, the user browses through pages of results sorted by the default ranking: $D_{1}\left(\mathbf{r}_{i}\right), \ldots, D_{k(h)}\left(\mathbf{r}_{i}\right)$, where $k(h) \geq 1$ is the number of such pages observed. About 60 percent of users in our sample do not go further in their search. At the second stage, the user employs search tools that produce an additional set $\tilde{\mathbf{C}}\left(h_{i}, P_{i}\right)$ of hotel results, which is not affected by ranking. We expect this simplification to have little effect, as it concerns only users who employ filtering tools.

Given the assumption of exogenous search preferences, the probability that a particular user will choose a search strategy $h$ can be approximated by its frequency among past users, $\rho_{h}$. Integrating over potential search strategies and unobserved tastes, we obtain the objective function of the ranking:

$$
\begin{gathered}
\operatorname{CTR}\left(P_{i}, R_{i}, \mathbf{r}_{i}\right)=\sum_{h} \rho_{h} \operatorname{CTR}\left(\mathbf{C}\left(h, P_{i}, \mathbf{r}_{i}\right), P_{i}, R_{i}\right) \\
=\sum_{h} \rho_{h}\left[\int \frac{\sum_{k \in \mathbf{C}\left(h, P_{i}, \mathbf{r}_{i}\right)} \exp \left(\mu_{i k}\right)}{\exp \left(\mu_{i 0}\right)+\sum_{k \in \mathbf{C}\left(h, P_{i}, \mathbf{r}_{i}\right)} \exp \left(\mu_{i k}\right)} d F\left(\alpha_{i}\right)\right] \longrightarrow \max _{\mathbf{r}_{i}}
\end{gathered}
$$

We further simplify this formula by discretizing the distribution of unobserved price sensitivity. Recall that the price sensitivity parameter $\alpha_{i}=\alpha_{0 i}+\alpha_{1}^{\prime} R_{i}$ includes an unobserved component $\alpha_{0 i}$ and the observed component that is related to parameters of request, $R_{i}$. We assume a negative 
log-normal distribution for the unobserved parameter: $\ln \left(-\alpha_{0 i}\right) \sim N\left(\bar{\alpha}_{0}, \sigma_{\alpha}\right)$. Assuming $\bar{\alpha}_{0}, \sigma_{\alpha}$ are known (e.g. estimated) we discretize this distribution by defining a grid of parameter values, $\alpha_{0 i} \in\left\{\alpha_{0}^{1}, \ldots, \alpha_{0}^{G+1}\right\}$, which covers 99 percent of the density. A consumer $i$ is said to be of type $g(i)=g$ if he belongs to an interval $\alpha_{0 i} \in\left(\alpha_{0}^{g}, \alpha_{0}^{g+1}\right)$.

With this discretization, the type of consumer $i$ is defined by a unique combination $\left(\alpha_{0}^{g(i)}, R_{i}\right)$, which in our model captures all relevant information about average tastes. Conditionally on $\left(\alpha_{0}^{g(i)}, R_{i}\right)$, the optimal ranking amounts to sorting hotels by decreasing mean utilities, which are computed as:

$$
\hat{\mu}_{i j}^{g(i)}=\left(\alpha_{0}^{g(i)}+\alpha_{1}^{\prime} R_{i}\right) p_{i j}+\left(\beta_{0}+\beta_{1}^{\prime} R_{i}\right) X_{j}
$$

Note that we have not included the position effect $\delta L_{i j}$ in the mean utility formula. We interpret the position effect as a reduced-form way to capture within-page search, not as an inherent part of utility. Below we discuss the identification the position effect.

Denote by $\mathbf{r}_{i}^{g(i)}$ the sorted list of mean utilities, $\hat{\mu}_{i j}^{g(i)}$. Because we do not observe $g(i)$, we cannot offer an optimal ranking to each consumer; at the same time, any ranking $\mathbf{r}_{i}^{g}, g \neq g(i)$, will result in click losses among consumers of a given type. Therefore, the objective is to "guess" the type of the current user, in a way that minimizes the click loss, given parameters of request $R_{i}$ and current prices $P_{i}$. With this approach, a generally intractable problem (10) reduces to a discrete optimization problem:

$$
\begin{gathered}
\operatorname{CTR}\left(P_{i}, R_{i}, \mathbf{r}_{i}^{g}\right)= \\
=\sum_{h} \rho_{h}\left[\sum_{g(i)=1}^{G} \phi_{g(i)} \frac{\sum_{k \in \mathbf{C}\left(h, P_{i}, \mathbf{r}_{i}^{g}\right)} \exp \left(\mu_{i k}^{g(i)}\right)}{\exp \left(\mu_{i 0}^{g(i)}\right)+\sum_{k \in \mathbf{C}\left(h, P_{i}, \mathbf{r}_{i}^{g}\right)} \exp \left(\mu_{i k}^{g(i)}\right)}\right] \rightarrow \max _{g}
\end{gathered}
$$

In this formula, the inner sum represents integration over unobserved consumer type, $g(i)$. The probability of each type, $\phi_{g(i)}=P\left(\alpha_{0 i} \in\left(\alpha_{0}^{g}, \alpha_{0}^{g+1}\right)\right)$, is computed using estimated density of the parameter $\alpha_{0 i}$. In computing the CTR, it is important to bring back the position effect, $\mu_{i k}^{g(i)}=\hat{\mu}_{i j}^{g(i)}+\delta L_{i j}$, as one of the determinants of click. Also note that because the ranking affects CTR only through the choice set mapping function $\mathbf{C}\left(h, P_{i}, \mathbf{r}_{i}^{m}\right)$, the set of type-specific rankings, $\mathbf{r}_{i}^{g(i)}$ can be pre-computed and stored in memory.

To summarize, the algorithm is the following: 
1. User $i$ submits a search request $R_{i}$.

2. Search engine receives information about $\left(P_{i}, R_{i}\right)$, as well as the set of available hotels.

3. For each available hotel and each possible unobserved type $g$, compute position-free mean utilities $\hat{\mu}_{i j}^{g}$.

4. Using results of Step 3, form a ranked list of hotels $\mathbf{r}_{i}^{g}$ for each unobserved type, $g=1, \ldots, G$.

5. For each ranking $\mathbf{r}_{i}^{g}$, compute $C T R\left(P_{i}, R_{i}, \mathbf{r}_{i}^{g}\right)$ using formula (12).

6. Choose the ranking that delivers the highest CTR.

All "deep" parameters of the model, such as consumer preferences, frequencies of search strategies, probabilities of consumer types, are pre-computed and stored in memory. The steps 1-5 that need to be repeated for each user are fast, as they involve only exponentiation, linear operations, and sorting. This ensures speed and scalability of computations, which are critical properties of the ranking system.

\subsection{Two Product Ranking Examples}

Given that consumer types are unobserved, it is not a priori clear how such information could be incorporated into a ranking, and why such a strategy may be better than a ranking based on average tastes. The following simple examples illustrate this issue, emphasizing the role of changing prices in the difference between two ranking approaches.

Suppose two products, characterized by price-quality combinations $\left(p_{j}, v_{j}\right), j=1,2$. Product 2 has twice the quality of product 1 , with $v_{2}=20>v_{1}=10$, but is also more expensive, with $p_{2}>p_{1}$. Product utilities are $u_{i j}=\alpha_{i} p_{j}+v_{j}+\varepsilon_{i j}, i=1,2$. Consumers can be of two types, according to their price sensitivity: let $\theta=1$ denote price sensitive consumers with $\alpha_{i}=-2$, and $\theta=2$ denote consumers with $\alpha_{i}=-1$. With these assumptions, if the price of the high quality product is is not too high, $p_{2}<p_{1}+10$, consumers of type 1 will prefer the cheap product, and consumers of type 2 , the expensive product. The share of type 1 in the population is $\phi=60 \%$.

All consumers observe the top ranked product; to find the second product, they need to search. Search can be done in two ways. With probability $\rho_{R}=50 \%$ a consumer will search default 
results, and with probability $\rho_{P}=20 \%$ she will sort by price and discover the cheapest result. The remaining $30 \%$ of consumers will not search.

Once search is over, some consumers will observe only one product, while others observe both. Denote by $D_{j}(\theta)$ the click rate among consumers of type $\theta$ whose choice set includes only product $j=1,2 .{ }^{14}$ Consequently, the expected click rate for is $\bar{D}_{j}=\phi D_{j}\left(\alpha_{1}\right)+(1-\phi) D_{j}\left(\alpha_{2}\right)$. Similarly, let $D_{12}\left(\alpha_{\theta}\right)$ be the CTR among consumers of type $\theta$ who observe both products, and $\bar{D}_{12}=$ $\phi D_{12}\left(\alpha_{1}\right)+(1-\phi) D_{12}\left(\alpha_{2}\right)$ be the expected CTR.

With this notation, we build two examples. In the first example, we abstract from heterogeneity in search preferences, and focus on the differences between the optimal ranking and average-taste ranking. In the second example, we reinstate the choice of search refinements and explore its implications for the optimal ranking.

\subsubsection{Comparison of Rankings under Random and Average Tastes}

Assume for the moment that the price sorting strategy is not available. Given that a portion $1-\rho_{R}$ of consumers will discover only the top ranked products, the click rate under ranking $\{1,2\}$ is:

$$
C T R(1,2)=\left(1-\rho_{R}\right) \bar{D}_{1}+\rho_{R} \bar{D}_{12}
$$

And similarly for $\{2,1\}$. The ranking $\{1,2\}$ is optimal if it leads to higher expected CTR: $(1-$ $\left.\rho_{R}\right) \bar{D}_{1}+\rho_{R} \bar{D}_{12}>\left(1-\rho_{R}\right) \bar{D}_{2}+\rho_{R} \bar{D}_{12}$, or simply

$$
\bar{D}_{1}>\bar{D}_{2}
$$

Now consider a ranking that targets preferences of the average consumer, whose price sensitivity is $\bar{\alpha}=\phi \alpha_{1}+(1-\phi) \alpha_{2}$. The expected CTR under ranking $\{1,2\}$ is found as $C T R(1,2 \mid \bar{\alpha})=$

\footnotetext{
${ }^{14}$ Both expressions are familiar logit formulae:$$
D_{j}(\theta)=\frac{\exp \left(\alpha_{\theta} p_{j}+v_{j}\right)}{1+\exp \left(\alpha_{\theta} p_{j}+v_{j}\right)}, j=1,2
$$$$
D_{12}(\theta)=\frac{\exp \left(\alpha_{\theta} p_{1}+v_{1}\right)+\exp \left(\alpha_{\theta} p_{2}+v_{2}\right)}{1+\exp \left(\alpha_{\theta} p_{1}+v_{1}\right)+\exp \left(\alpha_{\theta} p_{2}+v_{2}\right)}
$$ 
$\left(1-\rho_{R}\right) D_{1}(\bar{\alpha})+\rho_{R} D_{12}(\bar{\alpha})$. Consequently, the product ranking $\{1,2\}$ is chosen if and only if:

$$
D_{1}(\bar{\alpha})>D_{2}(\bar{\alpha})
$$

Observe that the latter inequality holds if and only if $\bar{\alpha} p_{1}+v_{1}>\bar{\alpha} p_{2}+v_{2}$. In other words, catering to average consumer type amounts to sorting results by declining average utility.

The ranking rule (14) and (15) will generally be different, as click rate is a non-linear function of the parameter of heterogeneity $\alpha_{\theta}$. However, the magnitude of the discrepancy will vary with product prices, both in the absolute and relative terms.

Figure 2(a) plots ranking rules (14) and (15) in the space of product prices, $p_{1}, p_{2}$. The dotted area corresponds to combinations of prices where both rankings place the cheaper product first. Indeed, if the relative price of the high-quality product is too high, it makes sense to cater to the tastes of the low type.

There are two areas where rankings diverge. In the area with horizontal lines, the average-taste model recommends product 2 (high quality) for the top position, while the random-tastes model favors the cheap product. In the area with vertical lines, predictions are the opposite. The reason for divergence is this. The contribution of a particular consumer type to the total demand is determined by its population share (which does not change with prices) and its individual demand (which does change with prices). As prices change, so do relative contributions of consumer types, and the optimal ranking must adjust accordingly. For instance, if prices of both products are high, the relative gain from catering to the low type will be small (as its total demand is low under both rankings), so it becomes optimal to target the high type, even though it is less prevalent in the population. The model based on average tastes ignores these changes in the composition of demand and can present suboptimal rankings. The resulting click losses can be quite large depending on price levels (see Appendix for a comparison between points A, B and C on Figure 2(a))

Consider points $\mathrm{A}, \mathrm{B}$ and $\mathrm{C}$, which correspond to different price levels, as well as different relative prices (product 2 is relatively more expensive in point $\mathrm{C}$ than in $\mathrm{A}$ and $\mathrm{B}$ ). At all points relative prices of two products are such that every type prefers his own-type product to be on top. In order to analyze compare the different models we calculate the click losses for each consumer type, when that type faces a non-preferred ranking. For instance, at point A the low type will 
reduce its clicks by 31.18 percent (or 5.44 percentage points) if presented with (high, low) ranking, relative to the (low, high). In Point $\mathrm{C}$, consumers of low type react strongly to non-preferred ranking, with a relative click loss of 49.85 percent. However, at this point the absolute loss in CTR is very low (only 0.08 percentage points) as the total demand is relatively small for low type consumers due to high prices. The opposite story holds for high types, as their demand drops at a much smaller rate as we move from A to C. Notice that as a result the random tastes model caters to low consumer types at point $\mathrm{A}$, and to high types at $\mathrm{B}$ and $\mathrm{C}$. The ranking based on average tastes overlooks these dynamics, presenting incorrect orderings in $\mathrm{A}$ and $\mathrm{C}$, where prices fall in the tails of the distribution.

\subsubsection{Ranking under Search Refinement}

Limited consumer search is the primary reason for the existence of ranking systems. Consumers do not explore all the options available to them. Further, they perform some optimal level of search of the options presented to them. Additionally they use filtering and sorting tools to explore additional options in the results. Chen and Yao (2012) find that consumers use these search refinement tools disproportionately if they are uninformed of the default ranking rule. As a result of the use of these tools, the same ranking will have heterogeneous impact on the choices of consumers who search differently.

An ideal ranking system will choose the contents of the first page in a way that complements future search results. For instance, if a consumer searches by price sorting, one may show more expensive hotels on the first page, thus increasing the diversity of the choice set. Unfortunately, individual search refinements are unobserved at the time when a ranking is to be made. Instead, we observe search refinements made by past consumers and their frequencies, much in the same way as we infer consumer types and their probabilities. Such distributional information can be a valuable input into the ranking method.

For an illustration, we return to our two-product example, where a proportion $\rho_{R}=50 \%$ of consumers will search the default results and discover the second-ranked result; alternatively, $\rho_{P}=20 \%$ consumers will sort by price and discover the cheapest result. We continue to assume two consumer types, where the low type always prefers the cheap product, and the high type always the expensive one. 
The expected CTR under the ranking $\{1,2\}$ is:

$$
\operatorname{CTR}(1,2)=\left(1-\rho_{D}-\rho_{P}\right) \bar{D}_{1}+\rho_{D} \bar{D}_{12}+\rho_{P} \bar{D}_{1}
$$

The first part is demand by consumers who did not search: their choice set consists of hotel 1 . The second part is demand by those who searched default results and so found both results. Finally, there are consumers who sorted by price and so did not find any new results, as the cheap product was already on the first page. the CTR under $\{2,1\}$ ranking is derived similarly. Taking the difference,

$$
C T R(1,2)-C T R(2,1)=\left(1-\rho_{D}-\rho_{P}\right)\left(\bar{D}_{1}-\bar{D}_{2}\right)-\rho_{P}\left(\bar{D}_{12}-\bar{D}_{1}\right)
$$

Suppose that $\bar{D}_{1}>\bar{D}_{2}$, i.e. consumers on average prefer the cheap product. As we have seen in the previous example, in the absence of price sorting this would be a sufficient condition to place the cheap product on top. Indeed, in that case the demand by non-searchers will gain $\left(\bar{D}_{1}-\bar{D}_{2}\right)$. However, in the presence of price sorting, consumers who search by price will lose $\left(\bar{D}_{12}-\bar{D}_{1}\right)$. The outcome of the tradeoff depends on the respective population shares of non-searchers, $\left(1-\rho_{D}-\rho_{P}\right)$ and price searchers, $\rho_{P}$, as well as the current prices.

Figure 2(b) illustrates this tradeoff by comparing the ranking rule (17) with a ranking that targets a consumer who only searches default results. Depending on price levels, two rankings may lead to divergent predictions, for two reasons. First, given that a portion of consumers will always discover the cheapest option, the expensive product gains in prominence (shaded area) under the ranking that accounts for price sorting. Second, consumers with different price sensitivities will benefit differently from the price sorting option. In point $\mathrm{A}$ in the figure, the price difference between products 1 and 2 is sufficiently small that any ranking would recommend placing product 2 on top. In point $\mathrm{B}$, the relative price of product 2 is much higher, and, if price sorting were not available, the cheap product would be ranked first unambiguously. With price sorting, it is still optimal to keep product 2 on top as the high consumer type who sort by price continue to benefit from this ranking. The price sorting tool increases the diversity of their choice sets. These gains outweigh the losses of low consumer types, who no longer buy product 2 (in point B it is too expensive). For them, it is preferable to put the cheap product on top, instead of relying on price 
sorting to locate it.

\section{Estimates of Consumer Choice}

The objective function for the choice of optimal ranking depends on the parameters of consumer utility, which are estimated on a dataset of clicks made by past consumers. In this way, we assume that a click is a revealed preference action, whereby a consumer indicates that a certain hotel is more attractive than other hotels in her choice set. This assumption is corroborated by previous studies, such as Joachims et al. (2005) and Brynjolfsson and Smith (2002) who compared click choices to actual purchases and found that clicks provide a reasonable proxy for the preferences of an average consumer.

There are three types of data that describe choices made by consumer $i$ on this website. First, $R_{i}$ is a vector of request parameters. Second, $C_{i}$ is a set of hotel indices observed by consumers during search. Third, let $j_{i}$ be the index of the clicked hotel, $j=0,1, \ldots, N$, where $N$ is the total number of hotels, with $j_{i}=0$ standing for the outside option of leaving the website without clicking. ${ }^{15}$ Conditional on these variables, the likelihood of an individual observation in our data is given by:

$$
P\left(j_{i} \mid C_{i}, P_{i}, R_{i}\right)=\int \frac{\exp \left(-\alpha_{i} p_{i j_{i}}+\beta_{i}^{\prime} X_{j_{i}}+\delta L_{i j_{i}}\right)}{\exp \left(\gamma_{0}+\gamma_{1}^{\prime} R_{i}\right)+\sum_{k \in C_{i}} \exp \left(-\alpha_{i} p_{i k}+\beta_{i}^{\prime} X_{k}+\delta L_{i k}\right)} d F\left(\alpha_{i}\right)
$$

where the distribution of individual taste parameters $\alpha_{i}, \beta_{i}$ is specified in (4) and (3).

Parameters to be estimated include: a) the effect of $R_{i}$ on the choice of outside option $\gamma_{0}, \gamma_{1}$; b) price sensitivity coefficients and a vector of tastes for non-price hotel features $\alpha_{0}, \alpha_{1}$ and $\beta_{0}, \beta_{1}$; and c) mean and variance of the unobserved heterogeneity in price sensitivity, $E\left(\alpha_{0 i}\right), V\left(\alpha_{0 i}\right)$. The estimation is performed by simulated maximum likelihood, where the integral over unobserved tastes is computed numerically at each step.

\footnotetext{
${ }^{15}$ Whenever several clicks are made, we take the last one as an endogenous outcome. Since the last click ends the search session, by that time the user has a more complete set of options, as compared to earlier clicks. For this reason, we interpret the last click as being more indicative of the user's preferences.
} 


\subsection{Identification}

The expression for individual likelihood (18) highlights the sources of variation that help identify consumer preferences: changing hotel prices, composition of choice sets, and positions of hotels on the display. In our data, these variations are unusually rich: for instance, no two users observed the same vector of hotel prices or had the same composition of choice set. By controlling for the choice set of a consumer, we greatly improve the precision of estimates without the need to rely on ad-hoc assumptions on the distribution of choice sets. ${ }^{16}$

With these data, it is possible to estimate more complex structures of unobserved heterogeneity than those implemented in our model. This should result in more flexible substitution patterns and better rankings. While being mindful of these potential benefits, we have chosen to limit our focus on heterogeneity in the price coefficient, as the hotel price is the only product characteristic that varies both across consumers and over time. Consequently, the primary challenge for a ranking system is to properly account for contemporaneous price information.

Identification of position effects is a separate challenge, because for a subset of observations the location of a hotel on a page is endogenous. If a click happened on a page that was sorted by default ranking, the position of that hotel is correlated with the past CTR and, consequently, with the hotel's unobserved quality. To separately identify the effect of position we need to use only variation in positions that is orthogonal to the variation in the hotel's quality over time. We proceed in the spirit of the control function approach: first, we regress a hotel's position $L_{i j}$ on a hotel's price and non-price characteristics, plus its CTR over the past two weeks; second, we take the residual from that regression and use it instead of actual $L_{i j}$ in the estimation of click preferences. There is also a substantial amount of exogenous variation in positions coming from displays of hotels that were sorted by price or distance to city center. For such position data, there is no correlation with unobserved quality because the ranking variable (price or distance) is explicitly controlled for.

\footnotetext{
${ }^{16}$ This property of the data is based on the ability of the web platform to record browsing activity by every visitor. The set of hotels observed during consumers' browsing or search session provides an approximation to the actual choice set. Arguably, the actual choice set may be larger, for example, due to prior consumer experience, or due to search of other information sources. However, hotel options not presented on the website only increase the value of the outside option and do not affect the relative probabilities of clicking on hotels within the choice set.
} 


\subsection{Estimation Results}

Table A-2 presents estimation results of two discrete choice models, a constant and a random coefficients model. To illustrate the economic magnitudes of these estimates, Table A-3 shows marginal effects of both models. The differences between the two models' marginal effects are substantial, which highlights the importance of including a random price coefficient, as we do in the second column. Below we will examine the implications of random tastes for the choice of optimal ranking and its outcome.

According to Table A-3, the median marginal effect of price is equal to -0.025 which means that if an average hotel price increases by 1 percent, the expected CTR drops by 0.025 percentage

points. Although this seems small, the extent of unobserved heterogeneity is substantial: the standard deviation of the marginal effect of price is 0.12 , which is 5 times the median.

In addition to unobserved heterogeneity, we include a rich set of interactions between consumer level observables - such as advance purchase, duration of stay, weekend stay and number of travelers - and both price and non-price hotel characteristics. For example, the least price sensitive are consumers who search two weeks in advance, while (perhaps contrary to expectation) the closer the arrival date is, the more price sensitive is demand.

Travelers who stay for more than two days are substantially less price sensitive than those who stay for a shorter period. Those who stay over a weekend are also slightly less price sensitive. We find similar results with respect to differences in tastes for star rating and distance to the city center. The magnitudes of these interaction terms are quite large (compared to the star rating coefficient, for instance). This implies that request parameters contain valuable information about consumer preferences that should be used by ranking systems.

Finally, the position effect estimates are economically plausible. Holding the hotel's quality constant, moving it down one position in list has the same effect on the expected CTR as increasing the hotel price by $\$ 2.13$. As there are 15 hotel displayed in the first page, this amounts to a $2.13 * 15$ $=\$ 32$ difference between the top and bottom positions on the initial page. 


\section{Evaluation of the Optimal Ranking}

There are two distinct ways in which the optimal ranking improves the quality of choice sets relative to the default ranking. The most important effect comes from changes in the composition of choice sets: what type of hotels will be displayed to consumer. Hotels that were available but neglected by the default ranking are introduced for consumers' consideration. At the same time, less relevant options are moved out of the choice set. Another effect is re-ordering hotels on the page of results: by putting better alternatives in better positions in the rankings, one can improve the quality of the choice set, even without changing its contents. This is particularly true for the first page of results, as it is observed by all users.

Ultimately, the evaluation of a optimal ranking must be made using controlled experiments, but in the absence of such possibility, we rely on the estimated model to evaluate its efficiency. For each consumer in the data, we generate a optimal ranking, and combine it with that consumer's search refinements to generate a counterfactual choice set. Conditional on that choice set, we use the discrete choice model to compute the expected CTR by that consumer under the optimal ranking. A similar calculation is made for the actual choice set that this consumer had under the default ranking. The increase in the CTR between the default and the counterfactual choice sets is the gain from the optimal ranking for this consumer. Repeating the exercise for each observation, we obtain the distribution of gains across consumers in our sample. Because we assume that the model of consumer choice is correct, there is necessarily an improvement in CTR for each user. Yet the comparison is not tautological, for two reasons.

First, although an improvement is necessarily obtained, its magnitude is not. As we show below, the magnitudes of the gains from optimal ranking are very large. As a robustness check, we simulated parameter values within one standard deviation of our current estimates, and re-ran the comparison (results are available upon request). We find that gains in CTRs remain substantial even under these perturbed parameters. This is not surprising, as our model incorporates economic factors that were shown to be important predictors of CTR: price of hotel, heterogeneity in tastes, and search refinements.

Second, our findings are corroborated by the model-free evidence of tracking positions of clicked hotels. We find that with our ranking, the majority of hotels that received a click improved their 
position: either moving to the first page or moving higher within the first page. In other words, consumers would have observed their preferred choice earlier, thus leading to improvements in CTRs.

\subsection{Tracking Click Positions}

In this exercise, we evaluate the optimal ranking by tracking positions of clicked hotels after the optimal ranking is applied. The premise is that consumers are better off if they find their preferred choices earlier. Given that clicked hotels are the preferred ones, an upward movement in position is a positive sign; in particular, movement of a clicked hotel from the second to the first page is bound to have strong impact.

Results are presented in Table 3. A total of 1,012 clicks were made by consumers either on the first page, or on subsequent pages of default results (clicks on hotels from refined search results are excluded, as they are not affected by ranking). Under the default ranking, only 141 (or 14 percent) of these clicks were made on the first page. Under the optimal ranking, this number increases to 636 clicks: when the optimal ranking was applied, 536 of additional clicked results were moved from beyond to the first page, an only 41 clicks were moved outside of the first page. In other words, under the optimal ranking, 63 percent of consumers would have found their preferred hotel without searching, as compared to 14 percent under the default ranking.

As an example, consider a two-star hotel, located in the O'Hare neighborhood of Chicago, 14 miles from the city center. It is a rarely clicked hotel (only 9 clicks), which prevents us from constructing a reliable CTR measure. Among the 9 users who clicked on it, the median position was originally \#29, or at the bottom of the second page (there are 15 hotels per page). Under the optimal ranking, the median position is \#11. For instance, for one consumer who clicked on this hotel, the position changed from \#94 (7th page of results) to \#11 (first page).

The evidence provided by the this experiment is robust, because modeling assumptions were used only for the construction of the ranking, but not for its evaluation. The limitation of these results is that they cannot be easily translated into CTR improvements. In the next section we discuss the improvements on the CTR from the optimal ranking. 


\subsection{Improvements in Click-through Rates}

For the purposes of optimal ranking, we discretize the distribution of unobserved heterogeneity. Specifically, we construct 40 potential consumer types, corresponding to different values of parameter $\alpha_{0 i}$, which stands for the unobserved component of the price sensitivity (see equation (4)). These values represent the grid points over the interval containing 99 percent of the probability mass of $\alpha_{0 i}$. For an economic interpretation, we compute price marginal effect for each type: the percentage point change in the expected CTR following a 1 percent increase in prices of all hotels. Figure 3(a) plots the resulting density of marginal effects: the median is around -1 percent, with substantial amount of heterogeneity around the median.

As discussed previously, the observable consumer-level heterogeneity in our sample consists of parameters of request, $R_{i}$, and a vector of prices, $P_{i}$. For every combination of $\left(R_{i}, P_{i}\right)$ observed in the sample and possible unobserved type $\alpha_{0}^{g}, g=1, \ldots, 40$, we construct a ranked list of hotels, denoted by vector $\mathbf{r}_{i}^{g}$, where each ranking is optimal conditional on the underlying unobserved type. For every ranking $\mathbf{r}_{i}^{g}$, we simulate counterfactual choice sets that would have been realized under this ranking. For every simulated choice set, an expected CTR is computed using the model's estimates. A ranking that delivers the highest CTR corresponds to a certain "guessed" consumer type $g_{i}^{*}=g^{*}\left(R_{i}, P_{i}\right)$, called the "best guess," which is the solution to the optimization problem (12). The notation $g^{*}\left(R_{i}, P_{i}\right)$ implies that the "best guess" will vary with prices and parameters of request.

Repeating the procedure for every observation, we obtain a sample distribution of "best guesses", $g_{i}^{*}, i=1, \ldots, M$, shown on Figure 3(b). The median type is most frequently chosen as the "best guess", but only in 30 percent of cases. In the remaining 70 percent of cases, types other than median are optimal for targeting. In other words, in the majority of cases a simple targeting of a median consumer leads to sub-optimal ranking. A further insight is obtained from Figure 4, which illustrates the effect of request parameters on the optimal group type that a consumer is assigned to. The figure shows the distribution of optimal "guesses" among users with different request parameters. The left figure shows a hypothetical request from "Guest 1" booking less than a week

in advance, and staying one night over a weekend; "Guest 2" is a hypothetical guest staying 3 or more nights, booking more than a week in advance, with no weekend stay. The figure shows that as 
these two hypothetical guests represent the two extremes of the price sensitivity distribution, the distribution of optimal group types differs greatly among them. For example, the right figure shows the typical "business guest" travels alone, stays 3 or more nights and does not include a weekend stay. The typical "leisure guest" books a room for two adults, spends one night, and includes a weekend stay. The figure shows that the most likely ranking of the typical business guest is the one for group 15 and the ranking for typical leisure guest is the ranking for group 17.

Table 4 presents expected CTRs under the default and optimal ranking. The impact on the optimal ranking is the highest for those users who only browsed the default search results (passive users). On average, the CTR increases from 29.71 percent in the default ranking to 71.27 percent, a 41.56 percentage point improvement. This improvement is due to both reordering and changing the composition of choice sets, because the counterfactual choice sets are based on evaluation of all currently available hotels. Using a consumer choice model, this improvement in CTRs translates into a 205 percent improvement in consumer welfare. ${ }^{17}$

Among all users, the improvement in the expected CTR is 38 percentage points, which is slightly lower, mostly due to a higher initial CTR among those who sort or filter search results. Indeed, we find that users who refined their search are more inclined to click, which makes sense because they are more likely to find a relevant hotel. Under the optimal ranking, CTRs are very similar across both groups of consumers, which is another illustration of the complementarity between ranking and search.

Figure 5 shows the sample density of CTRs, under the default and the optimal ranking. The density has multiple modes, which correspond to consumer types by search intensity. The dashed line in between represents CTR density under the optimal ranking that ignores unobserved heterogeneity in tastes. The benefit from this ranking is lower: the median CTR is around 40 percent, as opposed to 71 percent for the optimal ranking. This is because the average-taste model does not correctly capture the substitution between price and quality - a parameter that is of crucial importance in this context.

\footnotetext{
${ }^{17}$ It is worth noting that the computation of CTR under the proposed is not based on actual consumer choices that were used to identify consumer preferences: clicks and observed choice sets. We use only prices and request parameters, as well as empirical distribution of search strategies. For this reason, and because of the large sample size (relative to the number of parameters in the utility model), the prediction exercise does not suffer from the over-fitting problem.
} 


\section{Ranking under Endogenous Search}

\subsection{Search Model}

In this section we relax the assumption of exogenous consumer search and allow the ranking to affect how consumers search and what search refinement strategies they use. For instance, if preferred choices are observed earlier, there is less incentive for costly search. In this section, we estimate a model of consumer choice where both clicking and searching decisions are jointly determined.

When a consumer decides to search beyond the first page of results, her choice will depend on her preferences, expectations regarding the benefits of search, as well as search cost. Every consumer $i$ is endowed with an idiosyncratic search cost $c_{i}$, which is interpreted as a cognitive cost of exploring one page of results (containing 15 hotel options on this particular website). The distribution of search costs in the the population is assumed to be log-normal, whose mean and variance will be estimated. Let $C_{1 i}$ be the set of hotel results observed by consumer $i$ prior to search, and denote the utility of the best option as $U_{1 i}=\max \left\{u_{i j}, j \in C_{1 i}\right\}$. Further, let $\tilde{U}_{2}$ stand for the unknown best utility among search results; if search action $s$ is employed the distribution

of $\tilde{U}_{2}$ is $F_{s i}\left(\tilde{U}_{2}\right)$. Given search cost $c_{i}$, consumer will decide to search if and only if expected utility gain exceeds search cost:

$$
\int_{U_{1 i}}\left(\tilde{U}_{2}-U_{1 i}\right) d F_{s i}\left(\tilde{U}_{2}\right)>c_{i}
$$

Alternatively, this search rule can be stated in terms of reservation utility as:

$$
U_{1 i}<r_{s}\left(c_{i}\right)
$$

where $r_{s i}\left(c_{i}\right)$ is the solution to equation $\int_{r}\left(\tilde{U}_{2}-r\right) d F_{s i}\left(\tilde{U}_{2}\right)-c_{i}=0$.

Expectations regarding the search results depend on the type of search action: for instance, if $s$ stands for "price sorting", then the consumer expects to see the hotels ordered from the lowest to the highest price as a result, although the exact contents of that page are unknown. We represent consumer beliefs regarding the search results as an empirical distribution of search results observed by consumers who employed the same search action. Consumer preferences, given by equations (1), (2), (3) and (4), provide a mapping from the empirical distribution of search results into the 
utility space.

Although the large number of possible search refinement strategies - combinations of sorting and filtering tools - makes modeling this search activity challenging, we can limit the scope by focusing on the most relevant strategies. Table A-4 presents the distribution of search refinement strategies used by consumers in the sample. The most popular search strategy is to flip pages of default results, employed by 13.1 percent of users. Due to the popularity of such strategies, and also their importance for the effectiveness of ranking systems, we model every search decision made along the way. To do so, we adopt a fixed-sample search model: the user decides on the duration of search in advance and then executes that decision. For other strategies, we model only the first search action.

In our model, search unfolds in two stages. First, the consumer compares search strategies by their reservation utilities, $r_{1}\left(c_{i}\right), \ldots, r_{S}\left(c_{i}\right)$, and chooses the one with the highest value. From Table A-4, we infer that the consumer has 21 alternatives at this stage: 5 search actions that explore default results and 16 refinements that employ sorting/filtering tools. We use multinomial logit to model the choice at this stage. That is, the probability of user $i$ choosing strategy $s^{*}$ is:

$$
P\left(s^{*} \mid c_{i}\right)=\frac{\exp \left(r_{s^{*}}\left(c_{i}\right)\right)}{\sum_{s} \exp \left(r_{s}\left(c_{i}\right)\right)}
$$

For users who searched, the value of $s^{*}$ is observed; for non-searchers, $s^{*}$ needs to be integrated out. On the second stage, the user compares the value of the first page, $U_{1 i}$, with the reservation utility of the chosen strategy, $r_{s^{*}}\left(c_{i}\right)$, and decides whether to search in this way (see equation (20)). Finally, the user decides which hotel to click on among those in her choice set, $C_{i}$. If the user did not search, her choice set consists only of first-page hotels, $C_{i}=C_{1 i}$ (which includes the outside option); otherwise the set of search results is added: $C_{i}=C_{1 i}+C_{2 i}$. We continue to assume that a click is a revealed preference action, that is hotel $j^{*}$ gets clicked only if:

$$
u_{i j^{*}}>u_{i j}, \forall j \in C_{i}
$$

Accordingly, there are three endogenous variables in the model: a) choice of search strategy, b) search decision, and c) click decision. Integrating out inequalities (20) and (22) over unobserved 
utility shocks, we obtain likelihoods of searching and clicking decisions conditional on the chosen search strategy and search cost. There are three types of observations: a) consumers who did not search; b) consumers who searched but returned and clicked on the first page; and c) consumers who searched and clicked among the search results. Their likelihoods are, correspondingly:

$$
\begin{aligned}
P\left(\text { no search }, j^{*} \in C_{1 i} \mid s^{*}, c_{i}\right) & =L\left(j^{*}, C_{1 i}\right)\left(1-F\left(r_{s^{*}}\left(c_{i}\right), C_{1 i}\right)\right) \\
P\left(\text { search }, j^{*} \in C_{1 i} \mid s^{*}, c_{i}\right) & =L\left(j^{*}, C_{i}\right)\left(1-F\left(r_{s^{*}}\left(c_{i}\right), C_{i}\right)\right) \\
P\left(\text { search }, j^{*} \in C_{2 i} \mid s^{*}, c_{i}\right) & =L\left(j^{*}, C_{i}\right)\left(1-F\left(r_{s^{*}}\left(c_{i}\right), C_{i}\right)\right) \\
& +L\left(j^{*}, C_{2 i}\right) F\left(r_{s^{*}}\left(c_{i}\right), C_{1 i}\right)\left(1-F\left(r_{s^{*}}\left(c_{i}\right), C_{2 i}\right)\right)
\end{aligned}
$$

where $L(j, C)=\exp \left(\mu_{j}\right) / \sum_{k \in C} \exp \left(\mu_{k}\right)$, and $F(r, C)=\exp \left(-\sum_{k \in C} \exp \left(-\left(r-\mu_{k}\right)\right)\right)$. Unconditional likelihoods are obtained by multiplying these expressions by the choice probability (21):

$$
\begin{aligned}
P\left(\text { no search }, j^{*} \mid c_{i}\right) & =\sum_{s} P\left(\text { no search }, j^{*} \mid s, c_{i}\right) P\left(s \mid c_{i}\right) \\
P\left(\text { search }, s^{*}, j^{*} \mid c_{i}\right) & =P\left(\text { search }, j^{*} \mid s^{*}, c_{i}\right) P\left(s \mid c_{i}\right)
\end{aligned}
$$

The estimation is done by simulated maximum likelihood, where expressions (26), (27) are numerically integrated over draws of search costs. Estimation results are shown in Table A-5. The specification of preferences is given by equations (1) to (4), except for the random price coefficientto save on computation time, we assumed it to be constant (as opposed to the static model, the search model with non-random price coefficient does not suffer from the IIA property). The estimates of the matching static discrete choice model can be found in the "constant coefficient" column of Table A-2, which for convenience we reproduce in Table A-5. There are statistically significant differences in estimates of the static discrete choice model and search model. This is explained by the fact that the static model ignores the endogeneity of search decisions, thus assuming that choice sets are exogenous; from the search model's perspective, choice sets are endogenous and correlated with preferences (the extent of search depends on both preferences and search costs). 


\subsection{Optimal Ranking and Search}

The optimal ranking in a search model shares most characteristics of the static discrete choice model, with one addition that search probabilities themselves are affected by ranking. Indeed, if consumers find better results earlier, they are less likely to search. Formally, the aggregate CTR in a search model is obtained by integrating out all future search decisions, conditional on the status quo, i.e. contents of the first page. As these contents change with the optimal ranking, the future search paths are affected accordingly. Model likelihoods (26), (27) are combined to derive the probability of click on any hotel:

$$
C T R_{i}=1-\left[P\left(\text { no search }, j^{*}=0 \mid c_{i}\right)+\sum_{s^{*}} P\left(\operatorname{search}, s^{*}, j^{*}=0 \mid c_{i}\right)\right]
$$

where $j^{*}=0$ indicates the choice of the outside option. Note that this CTR still depends on consumer-level unobservables (search cost, tastes) that need to be integrated out numerically. The expression (28) is calculated for alternative rankings, and the ranking with the highest value is chosen. Comparing the outcome with the CTR resulting from the default ranking, we evaluate the improvement.

Previously we have considered improvements in CTR conditionally on the chosen search strategy, assuming that the optimal ranking would not affect search. This assumption generally overstates the actual improvements, because the endogeneity of search decisions adds an opposite effect: with a better ranking, consumers will search less and end up with smaller choice sets. Other things equal, the discrete choice model predicts that a smaller choice set results in smaller-than-expected CTR. To evaluate this effect, we now consider ex-ante improvements in CTR, e.g., before the search refinements are made. Unfortunately, we can use only a small subset of data for these purposes, as we need to observe the default ranking under counterfactual search actions (as required by formula (28)). Such data is available only for 240 consumers who flipped five pages of default results: for these users, we observe default ranking under any search actions (no search, one page, two pages, etc.).

Table 5 presents CTR improvements from three different scenarios. The model with endogenous search results in a CTR improvement of 19.2 percentage points (from 35.5 to 54.7 percent) compared to the default ranking. In the model with endogenous search, the CTR increases from 
35.5 percent under the default ranking to 50 percent under the optimal ranking. This is smaller than 19.2 percentage points because the ranking was chosen under the assumption of exogenous search decisions, and also because static model estimates would be biased if consumers' search process is affected significantly by the ranking presented to them. However, these appear to be second-order effects: the ranking based on a simplified, static model brings $14.5 / 19.2=75$ percent of the improvement obtained in a more complex search model.

Additionally, on the third line we calculate CTRs under the assumption of exogenous search probabilities. The improvements are in line with those found in Table 4, and are much larger than those obtained under the endogenous search. This finding suggests that the optimal ranking should have an important effect on search refinements.

\section{Conclusions}

This paper presents an alternative ranking for online platforms using all the information available to the platform at the time of the request. We measure the sub-optimality of the default ranking derived from the persistence of popularity rankings that do not fully take into account price changes. A second reason for sub-optimality is the feedback property of the popularity ranking, as hotels that are shown on the first pages are also more likely to be chosen by the consumer. We show that the default ranking, based on popularity, exhibits these properties. We propose a consumer-preference based ranking that has the advantage of evaluating hotel choices at the time of request and as such accounts for changes in product attributes, such as price, that greatly affect consumer preference for the option but are not captured by a lagged popularity ranking. The counterfactual estimations indicate that the proposed model increases the CTR from 50 to 100 percent. Consumer benefits derive from more advantageous positions of preferred choices.

It is worth noting that the structural approach allows us to link a choice with the different consideration sets observed by the consumer as part of her search process. This modeling approach also allows for click prediction under alternative rankings and changing market conditions (availability and prices). This is difficult to achieve with reduced-form approaches to click, such as simple popularity rankings or more generally, machine-learning algorithms. Formally, the click maximization is achieved by improving the expected values of choice sets presented to consumers. 
The optimal ranking is computed for every individual consumer request, while conditioning on the set of currently available products and their prices. In this way, the proposed algorithm employs technological capacities of a search platform: its ability to collect information and manipulate displays in real time.

This paper has important managerial implications. Online platforms offer consumers the ability to analyze and compare product attributes for a large set of distinct alternative products. Incorporating consumer choice models that allow for heterogeneous tastes on a variety of attributes lets companies more precisely present consumers with preferred alternatives. In addition to increased profitability through higher CTRs, reducing search frictions will likely increase consumer satisfaction, as well as retention and return rates. 


\section{References}

Anderson, M., M. Ball, H. Boley, S. Greene, N. Howse, D. Lemire, and S. Mcgrath (2003): "RACOFI: A Rule-Applying Collaborative Filtering System," Working paper.

Ansari, A., S. Essegaier, and R. Kohli (2000): "Internet Recommendation Systems," Journal of Marketing Research, 37(3), 363-375.

Ansari, A., And C. F. Mela (2003): "E-Customization," Journal of Marketing Research, 40(2), $131-145$.

Basilico, J., And T. Hofmann (2004): "Unifying collaborative and content-based filtering," in In ICML, pp. 65-72. ACM Press.

Belanger, F. (2005): "A conjoint analysis of online consumer satisfaction," Journal of Electronic Commerce Research, 6, 95-111.

Berman, R., and Z. Katona (2011): "The Role of Search Engine Optimization in Search Marketing," Working paper.

Billsus, D., and M. J. Pazzani (1998): "Learning Collaborative Information Filters," in ICML '98: Proceedings of the Fifteenth International Conference on Machine Learning, pp. 46-54, San Francisco, CA, USA. Morgan Kaufmann Publishers Inc.

Bucklin, R. E., J. M. Lattin, A. Ansari, D. Bell, E. Coupey, S. Gupta, J. D. C. Little, C. Mela, A. Montgomery, and J. Steckel (2002): "Choice and the Internet: From Clickstream to Research Stream," Marketing Letters, 2002, 245-258.

Burke, R. (2000): "Knowledge-based recommender systems," Encyclopedia of Library and Information Science, 69(32), 180-200.

Canny, J. (2002): "Collaborative filtering with privacy via factor analysis," in SIGIR '02: Proceedings of the 25th annual international ACM SIGIR conference on Research and development in information retrieval, pp. 238-245, New York, NY, USA. ACM.

Chen, Y., And S. Yao (2012): "Search with Refinement," Working paper.

Ghose, A., P. Ipeirotis, And B. Li (2012): "Designing Ranking Systems for Hotels on Travel Search Engines by Mining User-Generated and Crowd-Sourced Content," Marketing Science, $31(3), 492-520$.

Ghose, A., And S. Yang (2009): "An Empirical Analysis of Sponsored Search in Online Advertising," Management Science, 55(10), 1605-1622.

Hendricks, K., A. Sorensen, and T. Wiseman (2010): "Observational Learning and Demand for Such Goods," Working paper.

Ho, D., And K. Imai (2006): "Randomization Inference with Natural Experiments: An Analysis of Ballot Effect in the 2003 California Recall Election," Journal of the American Statistical Association, 101, 888-900.

Hofmann, T. (1999): "Latent Class Models for Collaborative Filtering," in In Proceedings of the Sixteenth International Joint Conference on Artificial Intelligence, pp. 688-693. 
Huang, Z., D. D. Zeng, and H. Chen (2004): "A unified recommendation framework based on Probabilistic Relational Models," in In Fourteenth Annual Workshop on Information Technologies and Systems (WITS), pp. 8-13.

Joachims, T., L. Granka, B. Pan, H. Hembrooke, and G. Gay (2005): "Accurately Interpreting Clickthrough Data As Implicit Feedback," in SIGIR '05 Proceedings of the 28th Annual International ACM SIGIR Conference on Research and Development in Information Retrieval.

Kim, B.-D., And S.-O. KIM (2001): "A New Recommender System To Combine Content-Based And Collaborative Filtering Systems," Journal of Database Marketing, 8, 224.

Lohse, G. (1997): "Consumer Eye Movement Patterns on Yellow Pages Advertising," Journal of Advertising, 26(1), 61-73.

Melville, P., R. J. Mooney, and R. Nagarajan (2001): "Content-Boosted Collaborative Filtering," in In Proceedings of the 2001 SIGIR Workshop on Recommender Systems.

Moon, S., and G. J. Russell (2008): "Predicting Product Purchase from Inferred Customer Similarity: An Autologistic Model Approach," Management Science, 54(1), 71-82.

Urbany, J., P. Dickson, and A. Sawyer (2000): "Insights into Cross- and Within-Sotre Price Search: Retailers Estimates vs Consumer Self-Reports," Journal of Retailing, 76(2), 243-258.

Vozalis, M., and K. G. Margaritis (2008): "Unison-CF: A Multiple-component, Adaptive Collaborative Filtering System," Working paper.

Yu, K., A. Schwaighofer, and V. Tresp (2003): "Collaborative ensemble learning: Combining collaborative and content-based information filtering via hierarchical bayes," in In Proceedings of the 19th Conference on Uncertainty in Artificial Intelligence, pp. 616-623. 
Figure 1: Clicks by Page Reached and Position

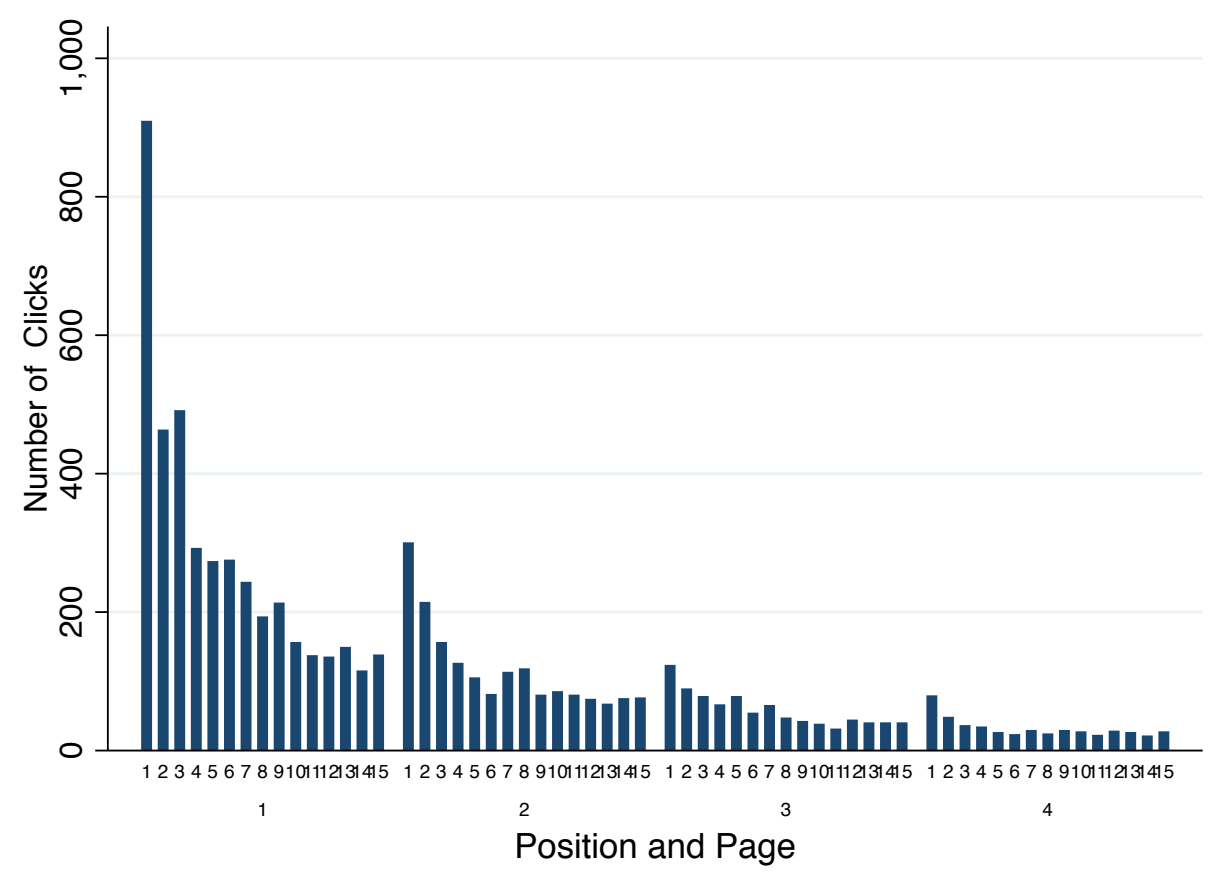

Note: The figure shows a breakdown of clicks by position and page where the click occurred. There are 15 results per page. The figure is truncated to the first four pages.

Figure 2: Two Product Ranking Examples

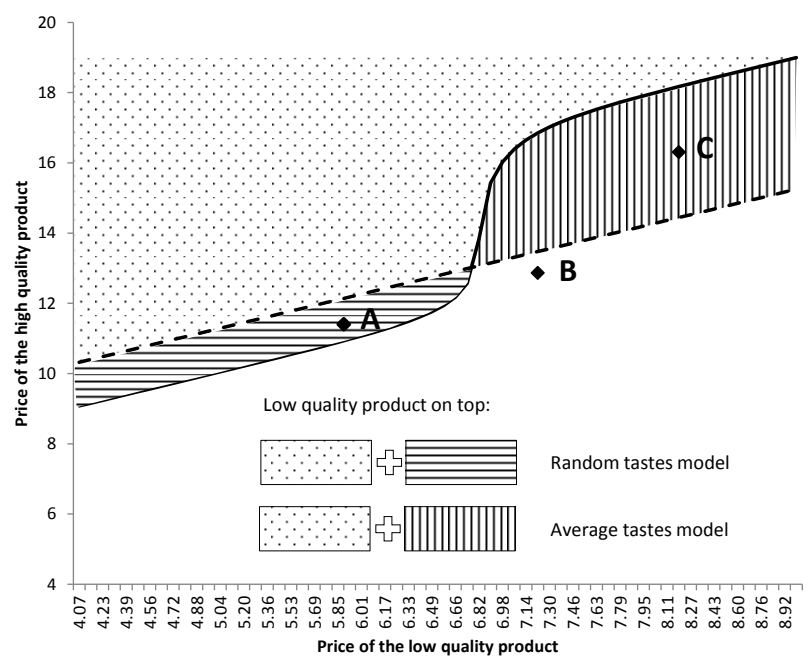

(a) No Search

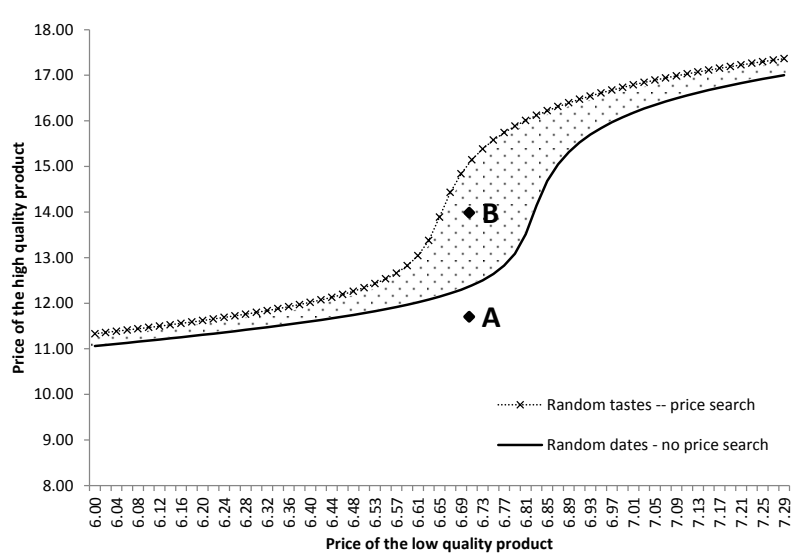

(b) Price Search 
Figure 3: Marginal Effects and Consumer Type

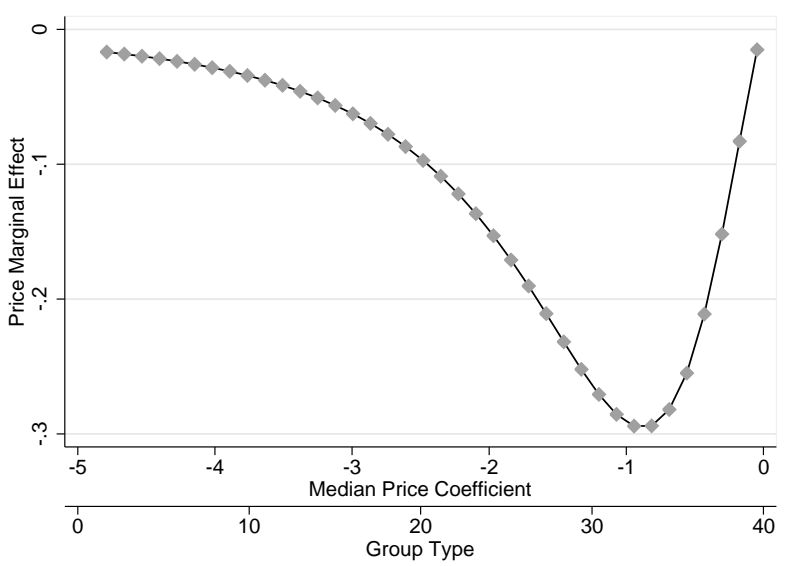

(a) Price Marginal Effects and Consumer Type

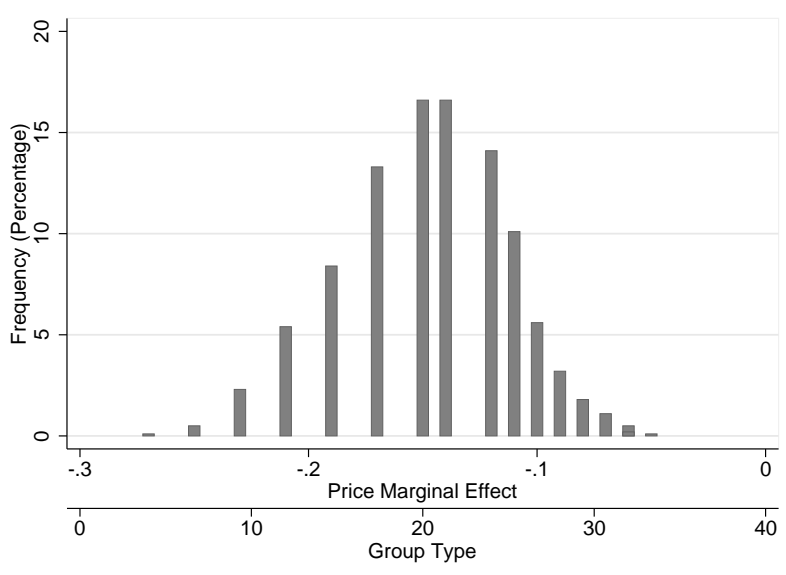

(b) Sample Frequency of "Best Guesses"

Figure 4: Sample Frequencies of "Best Guess" by Request Parameters

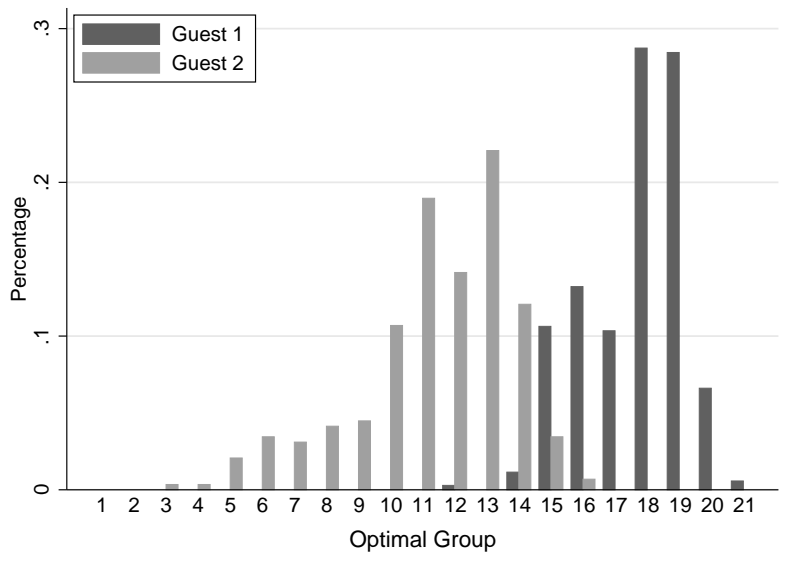

(a) Two Users with Extreme Price Elasticities

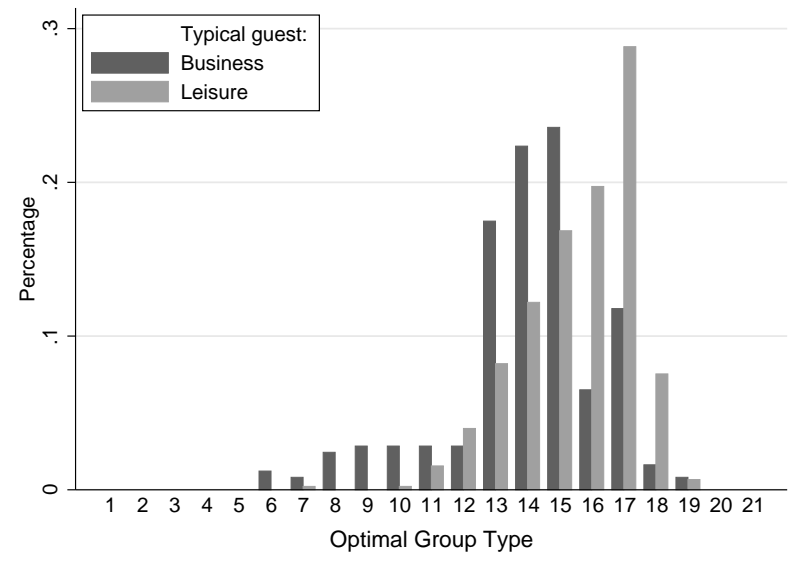

(b) Two Typical Users

Note: The figure shows the distribution of optimal "guesses" among users with different request parameters. On the left figure, "Guest 1" represents all users booking less than a week in advance, and staying one night over weekend; "Guest 2" represents users staying 3 or more nights, booking more than a week in advance, with no weekend stay. On the right figure, the typical "Business guest" travels alone, stays 3 or more nights and does not include a weekend stay. The typical "Leisure guest" consists of two adults, spending one night, and including a weekend stay. 
Figure 5: Sample Distributions of CTRs under Old and Optimal Ranking

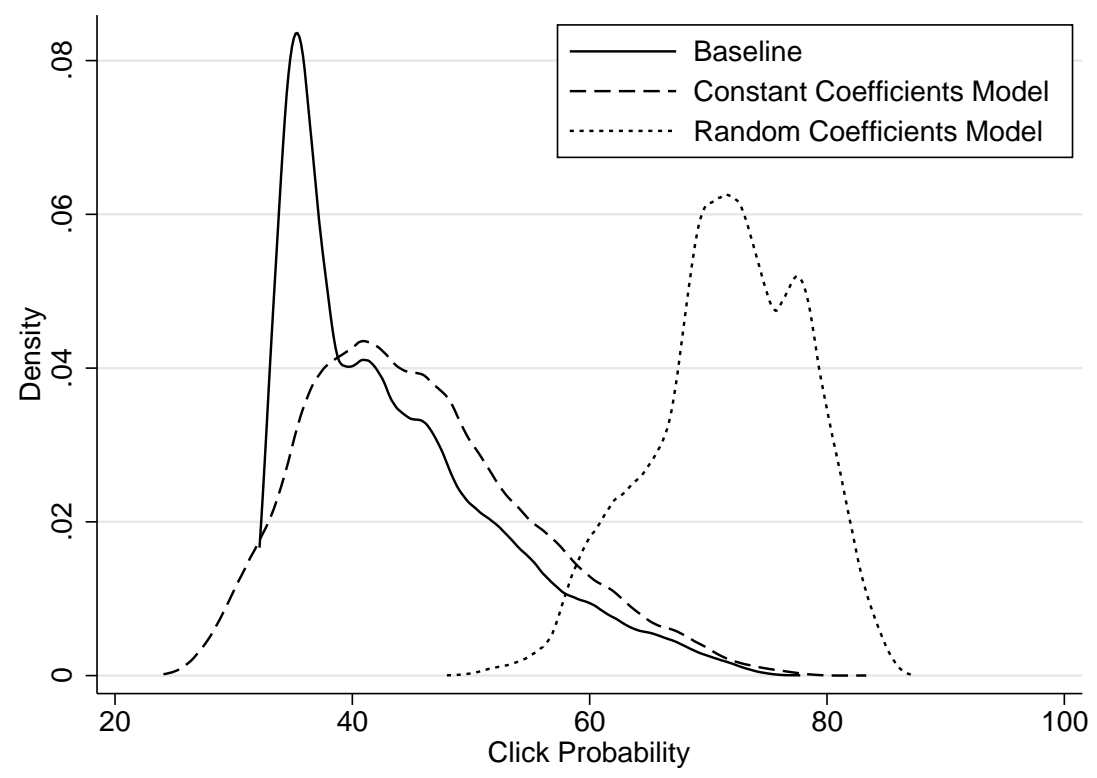

Table 1: Descriptive Statistics

\begin{tabular}{lrrrrrr}
\hline \hline & Mean & Median & Std. Dev. & Min. & Max. & Obs. \\
\hline Request parameters & & & & & & \\
Advance search (days) & 33.50 & 21.00 & 36.63 & 1.00 & 364.00 & 23,959 \\
Weekend stay & 0.60 & 1.00 & 0.49 & 0.00 & 1.00 & 23,959 \\
Number of people & 1.84 & 2.00 & 0.97 & 1.00 & 8.00 & 23,959 \\
Length of stay (days) & 2.44 & 2.00 & 1.65 & 1.00 & 30.00 & 23,959 \\
& & & & & & \\
Across hotels & & & & & \\
First page location (\% of obs) & 30.64 & 22.18 & 28.33 & 0.00 & 98.35 & 148 \\
Price (100s of dollars) & 2.30 & 2.00 & 1.27 & 0.16 & 15.00 & 721,848 \\
Clicked Price (100s of dollars) & 2.00 & 1.75 & 1.17 & 0.26 & 14.41 & 8,007 \\
CTR (\%) & 1.11 & 0.80 & 0.94 & 0.00 & 5.82 & 148 \\
& & & & & & \\
Across consumers & & & & & & \\
Any click & 0.33 & 0 & 0.47 & 0 & 1 & 23,959 \\
Click on the first page & 0.17 & 0 & 0.38 & 0 & 1 & 23,959 \\
Size of choice set (\# of hotels) & 30.00 & 26.00 & 21.11 & 10.00 & 135.00 & 23,959 \\
\hline \hline
\end{tabular}


Table 2: Breakdown of Consumers by Search and Click Actions

\begin{tabular}{lcc}
\hline \hline Search category & \% of sample & \% of total clicks \\
\hline Browse default rankings & & \\
1 page & 40.8 & 36.3 \\
2 pages & 6.7 & 6.7 \\
3 pages & 4.4 & 4.2 \\
More than 3 pages & 5.7 & 4.8 \\
Use sorting or filtering tools & \\
2 pages or more & 42.4 & 48.0 \\
\hline Total & 100 & 100 \\
\hline \hline
\end{tabular}

Table 3: Position Improvements of Clicked Hotels

\begin{tabular}{lccc}
\hline \hline & First Page & Other Pages & Total \\
\hline Default Ranking & 141 & 871 & 1012 \\
Optimal Ranking & 636 & 376 & 1012 \\
\hline \hline
\end{tabular}

Table 4: Average Click-through Rates under Default and Optimal Ranking

\begin{tabular}{lcccc}
\hline \hline & Default ranking & Optimal ranking & Difference & Obs. \\
\hline Passive users & 29.71 & 71.27 & 41.56 & 10,659 \\
& $(9.22)$ & $(6.52)$ & $(9.30)$ & \\
All users & 32.74 & 71.58 & 38.83 & 19,978 \\
& $(9.88)$ & $(6.42)$ & $(9.89)$ & \\
\hline \hline
\end{tabular}

Note: The table presents average CTRs for the default ranking of the platform and the proposed optimal ranking. The rates are presented for users who do not use sorting or filtering tools (passive) browsing only the default results and all users.

Table 5: Ex-ante Improvements in CTR across Models

\begin{tabular}{llcc}
\hline \hline Ranking Model & Search & Default ranking (\%) & Optimal ranking (\%) \\
\hline Search & Endogenous & 35.5 & 54.7 \\
Logit & Endogenous & 35.5 & 50.0 \\
Logit & Exogenous & 29.5 & 64.0 \\
\hline \hline
\end{tabular}


Table A-1: Effects of Price and Parameters of Request on Pairwise Rankings

\begin{tabular}{lcc}
\hline \hline & OLS & Logit \\
& & Marg. Effects \\
Variable & $Y_{j k s}$ & $Y_{j k s}$ \\
\hline$P_{j s}-P_{k s}$ (in $\left.\$ 100 \mathrm{~s}\right)$ & 0.058 & 0.064 \\
& $(0.000)^{* * *}$ & $(0.001)^{* * *}$ \\
Weekend Stay & -0.003 & 0.006 \\
& $(0.001)^{* * *}$ & $(0.005)$ \\
Advance Search & 0.000 & 0.000 \\
& $(0.000)^{* * *}$ & $(0.000)$ \\
Number of Searchers & 0.000 & 0.000 \\
& $(0.000)^{* * *}$ & $(0.000)^{* * *}$ \\
\hline Sample & Full & Random \\
Hotel Fixed Effects & Yes & Yes \\
$R^{2}$ & 0.29 & \\
Log-likelihood & & $-26,067.32$ \\
Number of Observations & $3,124,288$ & 49,796 \\
\hline \hline
\end{tabular}

Note: The table presents the effects of prices and other request parameters on the relative position of pairs of hotels using a binary dependent variable $Y_{j k s}$, which takes the value of 1 if the position of hotel $j$ is lower than hotel $k$ and the value of 0 otherwise. For each user request, from the 148 hotels in the sample we constructed all the hotel pair combinations of the observed hotels resulting in 9,734 pairs. The explanatory variables include the price difference of the hotel pair, weekend stay dummy, the number of days in advance the hotel search is made, and the number of people searching that request and hotel dummy variables for each hotel. Standard errors in parentheses. ${ }^{*}$ significant at $10 \%,{ }^{*}$ significant at $5 \%,{ }^{* * *}$ significant at $1 \%$ 
Table A-2: Estimates of Discrete Choice Model of Hotel Choice

\begin{tabular}{|c|c|c|c|c|}
\hline \multirow{2}{*}{ Baseline price parameters } & \multicolumn{2}{|c|}{ "Constant Coefficient } & \multicolumn{2}{|c|}{ Random Coeffficent } \\
\hline & & & & \\
\hline Price $\left(\alpha_{0}\right)$ & -1.042 & $(0.029)^{* * *}$ & - & \\
\hline$E\left(\log \left(-\alpha_{0 i}\right)\right)$ & - & & 1.367 & $(0.055)^{* * *}$ \\
\hline$S D\left(\log \left(-\alpha_{0 i}\right)\right)$ & - & & 1.673 & $(0.074)^{* * *}$ \\
\hline \multicolumn{5}{|l|}{ Price and request interactions } \\
\hline Price \& one day advance & 0.088 & $(0.040)^{* *}$ & -0.339 & $(0.049)^{* * *}$ \\
\hline Price \& one week advance & 0.460 & $(0.044)^{* * *}$ & 0.068 & $(0.059)$ \\
\hline Price \& two weeks advance & 0.466 & $(0.047)^{* * *}$ & 0.184 & $(0.064)^{* * *}$ \\
\hline Price \& three weeks advance & 0.341 & $(0.054)^{* * *}$ & 0.106 & $(0.073)$ \\
\hline Price \& one day stay & -0.184 & $(0.026)^{* * *}$ & -0.380 & $(0.033)^{* * *}$ \\
\hline Price \& two days stay & -0.059 & $(0.024)^{* * *}$ & -0.169 & $(0.035)^{* * *}$ \\
\hline Price \& weekend stay & 0.174 & $(0.023)^{* * *}$ & 0.009 & $(0.029)$ \\
\hline \multicolumn{5}{|l|}{ Non-price characteristics } \\
\hline Number of stars & 0.305 & $(0.020)^{* * *}$ & 0.376 & $(0.022)^{* * *}$ \\
\hline Distance to city center & -0.396 & $(0.040)^{* * *}$ & -0.539 & $(0.042)^{* * *}$ \\
\hline Distance to airport & 0.319 & $(0.067)^{* * *}$ & 0.410 & $(0.068)^{* * *}$ \\
\hline Position (residualized, in dollars) & -6.038 & $(0.255)^{* * *}$ & -2.125 & $(0.212)^{* * *}$ \\
\hline \multicolumn{5}{|l|}{ Outside option and request interactions } \\
\hline \multicolumn{5}{|l|}{ Advance purchase } \\
\hline One day & 0.074 & $(0.116)$ & 0.502 & $(0.189)^{* * *}$ \\
\hline One week & -0.603 & $(0.128)^{* * *}$ & 0.102 & $(0.200)$ \\
\hline Two weeks & -0.856 & $(0.136)^{* * *}$ & -0.512 & $(0.202)^{* * *}$ \\
\hline Three weeks & -0.585 & $(0.155)^{* * *}$ & -0.236 & $(0.225)$ \\
\hline One day of stay & 0.131 & $(0.064)^{* *}$ & -0.121 & $(0.101)$ \\
\hline Two days of stay & 0.045 & $(0.060)$ & 0.072 & $(0.095)$ \\
\hline Weekend stay indicator & -0.345 & $(0.054)^{* * *}$ & -0.238 & $(0.083)^{* * *}$ \\
\hline One guest in the room & -0.327 & $(0.048)^{* * *}$ & -0.667 & $(0.133)^{* * *}$ \\
\hline Two guests in the room & -0.077 & $(0.046)^{*}$ & -0.098 & $(0.131)$ \\
\hline Requests and hotel characteristics interactions & & Yes & & Yes \\
\hline Neighbordhood indicators & & Yes & & Yes \\
\hline Month indicators & & Yes & & Yes \\
\hline Log-likelihood & & 39584.58 & & 8601.35 \\
\hline Number of observations & & 23959 & & 23959 \\
\hline
\end{tabular}

Note: The table presents estimates of a conditional logit model using consumer click on a given hotel offering as dependent variable. The random coefficient model uses a log-normal distribution for the price coefficient. Advance search is defined as the length of time (in days) between the search and the date of arrival. Default category for advance purchase: four weeks or more; for number of people: two persons or more; for number of days: three days or more. Standard errors in parentheses. ${ }^{*}$ significant at $10 \%,{ }^{* *}$ significant at $5 \%,{ }^{* * *}$ significant at $1 \%$. 
Table A-3: Marginal Effect Estimates of Hotel Choice on Requests, Position, and Hotel Characteristics

\begin{tabular}{|c|c|c|c|c|}
\hline & \multicolumn{2}{|c|}{ Constant coefficient } & \multicolumn{2}{|c|}{ Random coefficient } \\
\hline Price (median) & -0.018 & $(0.001)^{* * *}$ & -0.025 & $(0.006)^{* * *}$ \\
\hline Price (Std. Dev.) & & & 0.120 & $(0.016)^{* * *}$ \\
\hline Stars & 0.463 & $(0.040)^{* * *}$ & 0.587 & $(0.008)^{* * *}$ \\
\hline Distance to city center & -0.022 & $(0.002)^{* * *}$ & -0.098 & $(0.009)^{* * *}$ \\
\hline Distance to airport & 0.028 & $(0.006)^{* * *}$ & 0.038 & $(0.004)^{* * *}$ \\
\hline Position (residualized, in dollars) & -0.069 & $(0.003)^{* * *}$ & -0.036 & $(0.006)^{* * *}$ \\
\hline Advance purchase (days) & -0.001 & $(0.002)$ & 0.005 & $(0.009)$ \\
\hline Length of stay (days) & 0.074 & $(0.016)^{* * *}$ & 0.104 & $(0.003)^{* * *}$ \\
\hline Weekend stay indicator & 0.003 & $(0.005)$ & -0.028 & $(0.007)^{* * *}$ \\
\hline Number of people & 0.154 & $(0.031)^{* * *}$ & 0.113 & $(0.010)^{* * *}$ \\
\hline Price and request interactions & & Yes & & Yes \\
\hline Requests and hotel characteristics interactions & & Yes & & Yes \\
\hline Neighbordhood indicators & & Yes & & No \\
\hline Month indicators & & Yes & & No \\
\hline Log-likelihood & & 9584.58 & & 8601.35 \\
\hline Number of observations & & 23959 & & 23959 \\
\hline
\end{tabular}

Note: The table presents average marginal effects of hotel characteristics on expected CTR (measured in percentage points). Unless otherwise noted, marginal effects are reported as median. Standard errors in parentheses. ${ }^{*}$ significant at $10 \%,{ }^{* *}$ significant at $5 \%, * * *$ significant at $1 \%$. 
Table A-4: Distribution of Search Strategies by First Search Action

\begin{tabular}{|c|c|c|c|}
\hline$\#$ & Strategy & Observations & $\%$ of sample \\
\hline & Browse default rankings & & \\
\hline & Initial Page Only & 8,540 & 35.6 \\
\hline 1 & 1 additional page & 1,295 & 5.4 \\
\hline 2 & 2 additional page & 899 & 3.8 \\
\hline 3 & 3 additional page & 398 & 1.7 \\
\hline 4 & 4 additional page & 299 & 1.2 \\
\hline \multirow[t]{2}{*}{5} & 5 additional page & 240 & 1.0 \\
\hline & $\begin{array}{l}\text { Search Refinement Strategies } \\
\text { Sort by }\end{array}$ & & \\
\hline 1 & Increasing price & 2,507 & 10.5 \\
\hline 2 & Price and flip & 1,209 & 5.0 \\
\hline 3 & Distance to city center & 581 & 2.4 \\
\hline 4 & Distance and flip & 313 & 1.3 \\
\hline \multirow[t]{2}{*}{5} & Decreasing star rating & 287 & 1.2 \\
\hline & Filter by & & \\
\hline 6 & Price - max 200 & 518 & 2.2 \\
\hline 7 & Price $-\max 300$ & 281 & 1.2 \\
\hline 8 & Price $-\max 400$ & 222 & 0.9 \\
\hline 9 & Distance to city center -5 miles & 414 & 1.7 \\
\hline 10 & Distance to city center -2 miles & 266 & 1.1 \\
\hline 11 & Distance to city center -10 miles & 269 & 1.1 \\
\hline 12 & Landmark - O'Hare airport & 441 & 1.8 \\
\hline 13 & Landmark-Navy Pier & 250 & 1.0 \\
\hline 14 & Reset Landmark filters & 211 & 0.9 \\
\hline 15 & Neighborhood-Gold Coast & 269 & 1.1 \\
\hline \multirow[t]{3}{*}{16} & Neighborhood-Loop & 228 & 1.0 \\
\hline & Other search strategies & 4022 & 16.8 \\
\hline & Total & 23,959 & 100.0 \\
\hline
\end{tabular}


Table A-5: Estimates of Model with Endogenous Search

\begin{tabular}{|c|c|c|c|}
\hline Demand estimates & Logit model & Search & model \\
\hline Price (baseline category) & $-1.042 \quad(0.029)$ & -0.960 & $(0.026)$ \\
\hline Number of stars & $0.305 \quad(0.020)$ & 0.163 & $(0.019)$ \\
\hline Distance to city center & $-0.396 \quad(0.040)$ & -0.789 & $(0.038)$ \\
\hline Distance to airport & $(0.067)$ & 1.258 & $(0.064)$ \\
\hline Position effect (in $\$$ ) & $-6.038 \quad(0.255)$ & -13.351 & $(0.459)$ \\
\hline Search costs $(\$)$ & & & \\
\hline Median baseline & & 13.390 & $(0.554)$ \\
\hline Median cost of two pages & & 18.031 & $(0.443)$ \\
\hline Median cost of three pages & & 39.002 & $(1.399)$ \\
\hline Median cost of four pages & & 50.890 & $(2.040)$ \\
\hline Median cost of five pages & & 77.274 & $(3.174)$ \\
\hline Standard deviation of baseline & & 13.073 & $(2.282)$ \\
\hline Intercept effects of request parameters & Yes & \multicolumn{2}{|c|}{ Yes } \\
\hline Price and request parameters & Yes & \multicolumn{2}{|c|}{ Yes } \\
\hline Non-price characteristics and requests & Yes & \multicolumn{2}{|c|}{ Yes } \\
\hline Neighborhood dummies & Yes & \multicolumn{2}{|c|}{ Yes } \\
\hline Month of arrival dummies & Yes & \multicolumn{2}{|c|}{ Yes } \\
\hline Number of observations & 23959 & \multicolumn{2}{|c|}{19937} \\
\hline
\end{tabular}

